\title{
Physical properties of the planetary systems WASP-45 and WASP-46 from simultaneous multiband photometry
}

\author{
S. Ciceri, ${ }^{1 \star}$ L. Mancini, ${ }^{1,2 \star ~ J . ~ S o u t h w o r t h ~},{ }^{3}$ M. Lendl, ${ }^{4,5}$ J. Tregloan-Reed,,${ }^{3,6}$ \\ R. Brahm,,${ }^{7,8}$ G. Chen, ${ }^{9,10}$ G. D’Ago, ${ }^{11,12,13}$ M. Dominik, ${ }^{14}$ R. Figuera Jaimes, ${ }^{13,15}$ \\ P. Galianni, ${ }^{13}$ K. Harpsøe, ${ }^{16}$ T. C. Hinse, ${ }^{17}$ U. G. Jørgensen, ${ }^{15}$ D. Juncher, ${ }^{15}$ \\ H. Korhonen, ${ }^{15,18}$ C. Liebig, ${ }^{13}$ M. Rabus, ${ }^{1,7}$ A. S. Bonomo, ${ }^{2}$ K. Bott, ${ }^{19,20}$ \\ Th. Henning, ${ }^{1}$ A. Jordán, ${ }^{7,8}$ A. Sozzetti, ${ }^{2}$ K. A. Alsubai, ${ }^{21}$ J. M. Andersen, ${ }^{15,22}$ \\ D. Bajek, ${ }^{13}$ V. Bozza, ${ }^{12}$ D. M. Bramich,${ }^{20}$ P. Browne, ${ }^{13}$ S. Calchi Novati, ${ }^{11,12} \dagger, 23$ \\ Y. Damerdji, ${ }^{24}$ C. Diehl, ${ }^{25,26}$ A. Elyiv, ${ }^{23,27,28}$ E. Giannini, ${ }^{24}$ S-H. Gu, ${ }^{29,30}$ \\ M. Hundertmark,${ }^{15}$ N. Kains, ${ }^{31}$ M. Penny, ${ }^{15,32}$ A. Popovas, ${ }^{15}$ S. Rahvar, ${ }^{33}$ \\ G. Scarpetta, ${ }^{11,12,22}$ R. W. Schmidt, ${ }^{24}$ J. Skottfelt, ${ }^{15,34}$ C. Snodgrass, ${ }^{35,36}$ \\ J. Surdej, ${ }^{23}$ C. Vilela, ${ }^{3}$ X.-B. Wang ${ }^{28,29}$ and O. Wertz ${ }^{23}$ \\ Affiliations are listed at the end of the paper
}

Accepted 2015 November 16. Received 2015 November 11; in original form 2015 July 13

\begin{abstract}
Accurate measurements of the physical characteristics of a large number of exoplanets are useful to strongly constrain theoretical models of planet formation and evolution, which lead to the large variety of exoplanets and planetary-system configurations that have been observed. We present a study of the planetary systems WASP-45 and WASP-46, both composed of a mainsequence star and a close-in hot Jupiter, based on 29 new high-quality light curves of transits events. In particular, one transit of WASP-45 b and four of WASP-46 b were simultaneously observed in four optical filters, while one transit of WASP-46 b was observed with the NTT obtaining a precision of $0.30 \mathrm{mmag}$ with a cadence of roughly $3 \mathrm{~min}$. We also obtained five new spectra of WASP-45 with the FEROS spectrograph. We improved by a factor of 4 the measurement of the radius of the planet WASP- $45 \mathrm{~b}$, and found that WASP-46 b is slightly less massive and smaller than previously reported. Both planets now have a more accurate measurement of the density $\left(0.959 \pm 0.077 \rho_{\text {Jup }}\right.$ instead of $0.64 \pm 0.30 \rho_{\text {Jup }}$ for WASP-45 $\mathrm{b}$, and $1.103 \pm 0.052 \rho_{\text {Jup }}$ instead of $0.94 \pm 0.11 \rho_{\text {Jup }}$ for WASP-46 b). We tentatively detected radius variations with wavelength for both planets, in particular in the case of WASP-45 b we found a slightly larger absorption in the redder bands than in the bluer ones. No hints for the presence of an additional planetary companion in the two systems were found either from the photometric or radial velocity measurements.
\end{abstract}

Key words: planets and satellites: fundamental parameters - stars: fundamental parameters stars: individual: WASP-45 - stars: individual: WASP-46.

\section{INTRODUCTION}

The possibility to obtain detailed information on extrasolar planets, using different techniques and methods, has revealed some

\footnotetext{
*E-mail: ciceri@mpia.de (SC); mancini@mpia.de (LM)
}

$\dagger$ Sagan visiting fellow. unexpected properties that are still challenging astrophysicists. One of the very first was the discovery of Jupiter-like planets on very tight orbits, which are labelled hot Jupiters, and the corresponding inflation-mechanism problem (Baraffe, Chabrier \& Fortney 2014, and references therein). To find the answers to this and other open questions, it is important to have a proper statistical sample of exoplanets, whose physical and orbital parameters are accurately measured. 
One class of extrasolar planets, those which transit their host stars, has lately seen a large increase in the number of its known members. This achievement has been possible thanks to systematic transit-survey large programs, performed both from ground (TrES: Alonso et al. 2004; HATNet: Bakos et al. 2004; XO: McCullough et al. 2005; WASP: Pollacco et al. 2006; KELT: Pepper et al. 2007; MEarth: Charbonneau et al. 2009; HATSouth: Bakos et al. 2013; QES: Alsubai et al. 2013), and from space (CoRoT: Barge et al. 2008, Kepler: Borucki et al. 2011).

The great interest in transiting planets lies in the fact that it is possible to measure all their main orbital and physical parameters with standard astronomical techniques and instruments. From photometry, we can estimate the period, the relative size of the planet and the orbital inclination, while precise spectroscopic measurements provide a lower limit for the mass of the planet (but knowing the inclination from photometry the precise mass can be calculated) and the eccentricity of its orbit. Unveiling the bulk density of the planets allows the imposition of constraints on, or differentiation between, the diverse formation and migration theories which have been advanced (see Kley \& Nelson 2012; Baruteau et al. 2014 and references therein).

Furthermore, transiting planets allow astronomers to investigate their atmospheric composition, when observed in transit or occultation phases (e.g. Charbonneau et al. 2002; Richardson et al. 2003). However, is also important to stress that, besides instrumental limitations, the characterization of planets' atmosphere is made difficult by the complexity of their nature. Retrieving the atmospheric chemical composition may be hindered by the presence of clouds, resulting in a featureless spectrum (e.g. GJ 1214 b; Kreidberg et al. 2014).

In this work, we focus on two transiting exoplanet systems: WASP-45 and WASP-46. Based on new photometric and spectroscopic data, we review their physical parameters and probe the atmospheres of their planets.

\subsection{WASP-45}

WASP-45 is a planetary system discovered within the SuperWASP survey by Anderson et al. (2012) (hereafter A12). The light curve of WASP-45, shows a periodic dimming (every $P=3.126 \mathrm{~d}$ ) due to the presence of a hot Jupiter (radius $1.16_{-0.14}^{+0.28} \mathrm{R}_{\text {Jup }}$ and mass 1.007 $\pm 0.053 \mathrm{M}_{\text {Jup }}$ ) that transits the stellar disc. The host (mass of 0.909 $\pm 0.060 \mathrm{M}_{\odot}$ and radius of $\left.0.945_{-0.071}^{+0.087} R_{\odot}\right)$ is a $\mathrm{K} 2 \mathrm{~V}$ star with a higher metallicity than the $\operatorname{Sun}([\mathrm{Fe} / \mathrm{H}]=+0.36 \pm 0.12)$. The study of the $\mathrm{Ca}$ II $\mathrm{H}+\mathrm{K}$ lines in the star's spectra revealed weak emission lines, which indicates a low chromospheric activity.

\subsection{WASP-46}

WASP-46 is a G6 V-type star (mass $0.956 \pm 0.034 \mathrm{M}_{\odot}$, radius 0.917 $\pm 0.028 R_{\odot}$ and $\left.[\mathrm{Fe} / \mathrm{H}]=-0.37 \pm 0.13\right)$. A12 discovered a hot Jupiter (mass $2.101 \pm 0.073 \mathrm{M}_{\text {Jup }}$ and radius $1.310 \pm 0.051 \mathrm{R}_{\text {Jup }}$ ) that orbits the star every $1.430 \mathrm{~d}$ on a circular orbit. As in the case of WASP-45, weak emission visible in the $\mathrm{Ca}$ II $\mathrm{H}+\mathrm{K}$ lines indicates a low stellar activity. Moreover, the WASP light curve shows a photometric modulation, which allowed the measurement of the rotation period of the star, and a gyrochronological age of the system of 1.4 Gyr. By observing the secondary eclipse of the planet, Chen et al. (2014b) detected the emission from the day-side atmosphere finding brightness temperatures consistent with a low heat redistribution efficiency.

\section{OBSERVATIONS AND DATA REDUCTION}

A total of 29 light curves of 14 transit events of WASP-45 b and WASP-46 b, and five spectra of WASP-45 were obtained using four telescopes located at the ESO La Silla observatory, Chile. The details of the photometric observations are reported in Table 1.

The photometric observations were all performed with the telescopes in autoguided mode and out of focus to minimize the sources of noise and increase the signal-to-noise ratio $(\mathrm{S} / \mathrm{N})$. By spreading the light of each single star on many more pixels of the CCD, it is then possible to utilize longer exposure times without incurring saturation. Atmospheric variations, change in seeing, or tracking imprecision lead to changes in the position and/or size of the point spread function (PSF) on the CCD and, according to the different response of each single pixel, spurious noise can be introduced in the signal. By obtaining a doughnut-shaped PSF that covers a circular region with a diameter from roughly 15 to 30 pixels, the small variations in position are averaged out and have a much lower effect on the photometric precision (Southworth et al. 2009).

\section{2 m Euler-Swiss Telescope}

The imager of the $1.2 \mathrm{~m}$ Euler-Swiss telescope, EulerCam, is a 4000 $\times 4000 \mathrm{e} 2 \mathrm{v}$ CCD with a field of view (FOV) of $15.7 \times 15.7 \mathrm{arcmin}$, yielding a resolution of 0.23 arcsec per pixel. Since its installation in 2010, EulerCam has been used intensively for photometric followup observations of planet candidates from the WASP survey (e.g. Hellier et al. 2011; Lendl et al. 2014), as well as for the atmospheric study of highly irradiated giant planets (Lendl et al. 2013). We observed one transit of WASP-45 b and two transits of WASP-46 b with EulerCam between June and September 2011 using a Gunn $r$ ' fiter.

\section{$3.58 m N T T$}

One transit of WASP-46 b was observed with the New Technology Telescope (NTT) on 2011 October 23. The telescope has a primary mirror of $3.58 \mathrm{~m}$ and is equipped with an active optics system. The EFOSC2 instrument (ESO Faint Object Spectrograph and Camera 2), mounted on its Nasmyth B focus, was utilized. The field of view of its Loral/Lesser camera is $4.1 \times 4.1 \mathrm{arcmin}$ with a resolution of 0.12 arcsec per pixel. During the observation of the whole transit, only a small region of the CCD, including the target and some reference stars, was read out in order to diminish the readout time and increase the sampling. The filter used was a Gunn $g$ (ESO no. 782).

\subsection{4 m Danish Telescope}

Two transits of WASP-45 b and three of WASP-46 b were observed with the $1.54 \mathrm{~m}$ Danish Telescope, using the DFOSC (Danish Faint Object Spectrograph and Camera) instrument mounted at the Cassegrain focus. The instrument, now used exclusively for imaging, has a CCD with a FOV $13.7 \times 13.7$ arcmin and a resolution of 0.39 arcsec per pixel. The CCD was windowed and a Bessell $R$ filter was used for all the transits.

\section{2 m MPG Telescope - GROND}

The 2.2 m MPG Telescope holds in its Coudé-like focus the GROND (Gamma Ray Optical Near-infrared Detector) instrument (Greiner 
Table 1. Details of the transit observations presented in this work. $N_{\mathrm{obs}}$ is the number of observations, $T_{\exp }$ is the exposure time, $T_{\mathrm{obs}}$ is the observational cadence, and 'Moon illum.' is the fractional illumination of the Moon at the midpoint of the transit. The triplets of numbers in the Aperture radii column correspond to the inner radius of the circular aperture (star) and outer radii of the ring (sky) selected for the photometric measurements.

\begin{tabular}{|c|c|c|c|c|c|c|c|c|c|c|c|}
\hline Telescope & $\begin{array}{l}\text { Date of } \\
\text { first obs. }\end{array}$ & $\begin{array}{l}\text { Start time } \\
\text { (UT) }\end{array}$ & $\begin{array}{l}\text { End time } \\
\text { (UT) }\end{array}$ & $N_{\text {obs }}$ & $\begin{array}{l}T_{\exp } \\
\text { (s) }\end{array}$ & $\begin{array}{c}T_{\mathrm{obs}} \\
(\mathrm{s})\end{array}$ & Filter & Airmass & $\begin{array}{l}\text { Moon } \\
\text { illum. }\end{array}$ & $\begin{array}{l}\text { Aperture } \\
\text { radii }(p x)\end{array}$ & $\begin{array}{l}\text { Scatter } \\
\text { (mmag) }\end{array}$ \\
\hline \multicolumn{12}{|l|}{ WASP-45: } \\
\hline Eul $1.2 \mathrm{~m}$ & 2011 Nov 07 & $02: 12$ & $05: 57$ & 197 & 50 & 69 & Gunn $r$ & $1.00 \rightarrow 1.51$ & 89 per cent & $6.0^{a}$ & 0.91 \\
\hline Dan $1.54 \mathrm{~m}$ & 2012 Sep 02 & $05: 05$ & $08: 46$ & 122 & 100 & 106 & Bessel $R$ & $1.15 \rightarrow 1.51$ & 93 per cent & 176075 & 0.55 \\
\hline MPG $2.2 \mathrm{~m}$ & 2012 Oct 15 & $23: 40$ & 03:58 & 238 & 40 & 65 & Sloan $g^{\prime}$ & $1.46 \rightarrow 1.01$ & 1 per cent & 237085 & 0.85 \\
\hline MPG $2.2 \mathrm{~m}$ & 2012 Oct 15 & $23: 40$ & $03: 58$ & 240 & 40 & 65 & Sloan $r^{\prime}$ & $1.46 \rightarrow 1.01$ & 1 per cent & 235570 & 0.65 \\
\hline MPG $2.2 \mathrm{~m}$ & 2012 Oct 15 & $23: 40$ & $03: 58$ & 238 & 40 & 65 & Sloan $i^{\prime}$ & $1.46 \rightarrow 1.01$ & 1 per cent & 214565 & 0.83 \\
\hline MPG $2.2 \mathrm{~m}$ & 2012 Oct 15 & $23: 40$ & $03: 58$ & 241 & 40 & 65 & Sloan $z^{\prime}$ & $1.46 \rightarrow 1.01$ & 1 per cent & 175070 & 0.75 \\
\hline Dan 1.54 m & 2013 Jul 24 & $07: 47$ & $10: 43$ & 100 & 100 & 106 & Bessel $R$ & $1.15 \rightarrow 1.34$ & 97 per cent & 135570 & 0.62 \\
\hline \multicolumn{12}{|l|}{ WASP-46: } \\
\hline Eul $1.2 \mathrm{~m}$ & 2011 Jun 10 & 03:40 & $07: 11$ & 117 & 90 & 108 & Gunn $r$ & $1.95 \rightarrow 1.17$ & 63 per cent & $5.2^{1}$ & 1.26 \\
\hline Eul $1.2 \mathrm{~m}$ & 2011 Sep 01 & $02: 47$ & $06: 37$ & 72 & 170 & 180 & Gunn $r$ & $1.12 \rightarrow 1.36$ & 14 per cent & $4.3^{1}$ & 0.88 \\
\hline NTT $3.58 \mathrm{~m}$ & 2011 Oct 24 & $00: 42$ & $05: 24$ & 85 & 150 & 176 & Gunn $g$ & $1.14 \rightarrow 2.21$ & 10 per cent & 5280100 & 0.30 \\
\hline MPG $2.2 \mathrm{~m}$ & 2012 Jul 03 & $04: 19$ & $10: 29$ & 116 & 115 & 145 & Sloan $g^{\prime}$ & $1.12 \rightarrow 1.39$ & 100 per cent & 206580 & 0.56 \\
\hline MPG $2.2 \mathrm{~m}$ & 2012 Jul 03 & 04:19 & $10: 29$ & 121 & 115 & 145 & Sloan $r^{\prime}$ & $1.12 \rightarrow 1.39$ & 100 per cent & 226580 & 0.64 \\
\hline MPG $2.2 \mathrm{~m}$ & 2012 Jul 03 & $04: 19$ & $10: 29$ & 120 & 115 & 145 & Sloan $i^{\prime}$ & $1.12 \rightarrow 1.39$ & 100 per cent & 226580 & 0.56 \\
\hline MPG $2.2 \mathrm{~m}$ & 2012 Jul 03 & $04: 19$ & $10: 29$ & 119 & 115 & 145 & Sloan $z^{\prime}$ & $1.12 \rightarrow 1.39$ & 100 per cent & 236580 & 0.62 \\
\hline Dan $1.54 \mathrm{~m}$ & 2012 Sep 24 & $04: 24$ & 08:03 & 97 & 120 & 131 & Bessel $R$ & $1.14 \rightarrow 1.95$ & 66 per cent & 116580 & 1.52 \\
\hline MPG $2.2 \mathrm{~m}$ & 2012 Oct 17 & 01:03 & $05: 55$ & 106 & 90 & 116 & Sloan $g^{\prime}$ & $1.13 \rightarrow 2.27$ & 4 per cent & 2590105 & 0.80 \\
\hline MPG $2.2 \mathrm{~m}$ & 2012 Oct 17 & 01:03 & $05: 55$ & 107 & 90 & 116 & Sloan $r^{\prime}$ & $1.13 \rightarrow 2.27$ & 4 per cent & 2590105 & 0.75 \\
\hline MPG $2.2 \mathrm{~m}$ & 2012 Oct 17 & 01:03 & $05: 55$ & 109 & 90 & 116 & Sloan $i^{\prime}$ & $1.13 \rightarrow 2.27$ & 4 per cent & 2390100 & 0.81 \\
\hline MPG $2.2 \mathrm{~m}$ & 2012 Oct 17 & 01:03 & $05: 55$ & 108 & 90 & 116 & Sloan $z^{\prime}$ & $1.13 \rightarrow 2.27$ & 4 per cent & 2390105 & 0.85 \\
\hline MPG $2.2 \mathrm{~m}$ & 2013 Apr 25 & $06: 07$ & $10: 30$ & 59 & 120 to 170 & 215 & Sloan $g^{\prime}$ & $2.18 \rightarrow 1.15$ & 100 per cent & 246580 & 0.70 \\
\hline MPG $2.2 \mathrm{~m}$ & 2013 Apr 25 & 06:07 & $10: 30$ & 59 & 120 to 170 & 215 & Sloan $r^{\prime}$ & $2.18 \rightarrow 1.15$ & 100 per cent & 246580 & 0.63 \\
\hline MPG $2.2 \mathrm{~m}$ & 2013 Apr 25 & $06: 07$ & $10: 30$ & 57 & 120 to 170 & 215 & Sloan $i^{\prime}$ & $2.18 \rightarrow 1.15$ & 100 per cent & 256580 & 0.90 \\
\hline MPG $2.2 \mathrm{~m}$ & 2013 Apr 25 & 06:07 & $10: 30$ & 57 & 120 to 170 & 215 & Sloan $z^{\prime}$ & $2.18 \rightarrow 1.15$ & 100 per cent & 246580 & 0.81 \\
\hline MPG $2.2 \mathrm{~m}$ & 2013 Jun 17 & $05: 36$ & 09:01 & 83 & 130 to 70 & 137 & Sloan $g^{\prime}$ & $1.28 \rightarrow 1.12$ & 55 per cent & 267085 & 0.61 \\
\hline MPG $2.2 \mathrm{~m}$ & 2013 Jun 17 & $05: 36$ & 09:01 & 82 & 130 to 70 & 137 & Sloan $r^{\prime}$ & $1.28 \rightarrow 1.12$ & 55 per cent & 267085 & 0.64 \\
\hline MPG $2.2 \mathrm{~m}$ & 2013 Jun 17 & $05: 36$ & 09:01 & 84 & 130 to 70 & 137 & Sloan $i^{\prime}$ & $1.28 \rightarrow 1.12$ & 55 per cent & 286580 & 0.70 \\
\hline MPG $2.2 \mathrm{~m}$ & 2013 Jun 17 & $05: 36$ & 09:01 & 81 & 130 to 70 & 137 & Sloan $z^{\prime}$ & $1.28 \rightarrow 1.12$ & 55 per cent & 287085 & 0.85 \\
\hline Dan $1.54 \mathrm{~m}$ & 2013 Aug 06 & $07: 19$ & $10: 28$ & 99 & 100 & 115 & Bessel $R$ & $1.12 \rightarrow 1.53$ & 1 per cent & 116580 & 0.68 \\
\hline Dan $1.54 \mathrm{~m}$ & 2013 Aug 28 & 04:06 & 08:39 & 132 & 100 & 125 & Bessel $R$ & $1.15 \rightarrow 1.43$ & 42 per cent & 126580 & 0.88 \\
\hline
\end{tabular}

Note. ${ }^{a}$ The aperture radii of the Euler-Swiss light curves are referred to the target aperture and are expressed in arcsec.

et al. 2008). GROND is a seven channel imager capable of performing simultaneous observations in four optical bands $\left(g^{\prime}, r^{\prime}, i^{\prime}\right.$, $z^{\prime}$, similar to Sloan filters) and three near-infrared (NIR) bands $(J$, $H, K)$. The light, split into different paths using dichroics, reaches two different sets of cameras. The optical cameras have $2048 \times$ 2048 pixels with a resolution of 0.16 arcsec per pixel. The NIR cameras have a lower resolution 0.60 arcsec per pixel, but a larger FOV of $10^{\prime} \times 10^{\text {arcmin }}$ (almost double the optical ones). The primary goal of GROND is the detection and follow-up of the optical/NIR counterpart of gamma-ray bursts, but it has already proven to be a great instrument to perform multicolour, simultaneous, photometric observations of planetary-transit events (e.g. Mancini et al. 2013a; Southworth et al. 2015). With this instrument, we observed one transit event of the planet WASP-45 $b$ and four of WASP-46 b.

The exposure time must be the same for each optical camera, and is also partially constrained by the NIR exposure time chosen. We therefore decided to fix the exposure time to that optimizing the $r^{\prime}$-band counts (generally higher than in the other bands) in order to avoid saturation.

\section{2 m MPG Telescope - FEROS}

The 2.2 m MPG telescope also hosts FEROS (Fibre-fed Extended Range Optical Spectrograph). This échelle spectrograph covers a wide wavelength range of 370-860 $\mathrm{nm}$ and has an average resolution of $R=48000 \pm 4000$. The precision of the radial velocity (RV) measurements obtained with FEROS is $\operatorname{good}$ enough $\left(\geq 10 \mathrm{~m} \mathrm{~s}^{-1}\right)$ for detecting and confirming Jupiter-size exoplanets (e.g. Penev et al. 2013; Jones et al. 2015). Simultaneously to the science observations, we always obtained a spectrum of a Th-Ar lamp in order to have a proper wavelength calibration. Five spectra of WASP-45 were obtained with FEROS.

\subsection{Data reduction}

For all photometric data, a suitable number of calibration frames, bias and (sky) flat-field images, were taken on the same day as the observations. Master-bias and flat-field images were created by median-combining all the individual bias and flat-field images, and used to calibrate the scientific images.

With the exception of the EulerCam data, we then extracted the photometry from the calibrated images using a version of the aperture photometry algorithm DАОРНОТ (Stetson 1987) implemented in the DEFOT pipeline (Southworth et al. 2014). We measured the flux of the targets and of several reference stars in the FOVs, selecting those of similar brightness to the target and not showing any significant brightness variation due to intrinsic variability or instrumental effects. For each data set, we tried different aperture sizes for both 
the inner and outer rings, and the final ones that we selected (see Table 1) were those that gave the lowest scatter in the out-of-transit (OOT) region. Light curves were then obtained by performing differential photometry using the reference stars in order to correct for non-intrinsic variations of the flux of the target, which are caused by atmospheric or air-mass changes. Also in this case, we tried different combinations of multiple comparison stars and chose those that gave the lowest scatter in the OOT region. We noticed that the different options gave consistent transit shapes but had a small effect on the scatter of the points in the light curves. Finally, each light curve was obtained by optimizing the weights of the chosen comparison stars.

The EulerCam photomety was also extracted using relative aperture photometry with the extraction being performed for a number of target and sky apertures, of which the best was selected based on the final light-curve RMS. The selection of the reference stars was done iteratively, optimizing the scatter of the full transit light curves based on preliminary fits of transit shapes to the data at each optimization step. For further details, please see Lendl et al. (2012).

To properly compare the different light curves and avoid underestimation of the uncertainties assigned to each photometric point, we inflated the errors by multiplying them by the $\sqrt{\chi_{v}^{2}}$ (defined as $\chi_{v}^{2}=\Sigma\left(\left(x_{i}-x_{\text {best }}\right)^{2} / \sigma^{2}\right) /$ DOF, where DOF is the number of degrees of freedom) obtained from the first fit of each light curve. We then took into account the possible presence of correlated noise or systematic effects using the $\beta$ approach (e.g. Gillon et al. 2006; Winn et al. 2007), with which we further enlarged the uncertainties.

The NIR light curves observed with GROND were reduced following Chen et al. (2014a), by carefully subtracting the dark from each image and flat-fielding them, and correcting for the read-out pattern. No sky subtraction was performed since no such calibration files were available. Unfortunately, the quality of the data was not good enough to proceed with a detailed analysis of the transits.

\section{FEROS spectra reduction}

The spectra obtained with FEROS were extracted using a new pipeline written for échelle spectrographs, adapted for this instrument and optimised for the subsequent RV measurements (Jordán et al. 2014; Brahm et al. 2015). In brief, first a master-bias and a master-flat were constructed as the median of the frames obtained during the afternoon routine calibrations. The master-bias was subtracted from the science frames in order to account for the CCD intrinsic inhomogeneities, while the master-flat was used to find and trace all 39 échelle orders. The spectra of the target and the calibration Th-Ar lamp were extracted following Marsh (1989). The science spectrum was then calibrated in wavelength using the $\mathrm{Th}-\mathrm{Ar}$ spectrum, and a barycentric correction was applied. In order to measure the RV of the star, the spectrum was cross-correlated with a binary mask chosen according to the spectral class of the target. For each échelle order a cross-correlation function (CCF) was found and the RV measured by fitting a combined one, which is obtained as a weighted sum of all the CCF, with a Gaussian. The uncertainties on the RVs were calculated using empirical scaling relations from the width of the $\mathrm{CCF}$ and the mean $\mathrm{S} / \mathrm{N}$ measured around $570 \mathrm{~nm}$. The RV measurements are reported in Table 2.

\section{LIGHT-CURVE ANALYSIS}

The light-curve shape of a transit (its depth and duration) directly depends on values that describe the planet and its host star (e.g.
Table 2. FEROS RV measurements of WASP-45.

\begin{tabular}{ccc}
\hline $\begin{array}{c}\text { Date of observation } \\
\text { BJD-2400000 }\end{array}$ & $\begin{array}{c}\mathrm{RV} \\
\left(\mathrm{km} \mathrm{s}^{-1}\right)\end{array}$ & $\begin{array}{c}\mathrm{err}_{\mathrm{RV}} \\
\left(\mathrm{km} \mathrm{s}^{-1}\right)\end{array}$ \\
\hline 56939.69704138 & 4.368 & 0.010 \\
56941.64378526 & 4.557 & 0.011 \\
56942.69196252 & 4.382 & 0.010 \\
57037.53926492 & 4.650 & 0.010 \\
57049.54110894 & 4.502 & 0.010 \\
\hline
\end{tabular}

Table 3. Stellar atmospheric parameters used to calculate the LD coefficients used to model the light curves.

\begin{tabular}{lcc}
\hline Parameter & WASP-45 & WASP-46 \\
\hline$T_{\text {eff }}(K)$ & 5100 & 5600 \\
$\log g\left(\mathrm{~cm} \mathrm{~s}^{-2}\right)$ & 4.5 & 4.5 \\
{$\left[\frac{\mathrm{Fe}}{\mathrm{H}}\right]$} & 0.5 & -0.3 \\
$V_{\text {micro }}\left(\mathrm{km} \mathrm{s}^{-1}\right)$ & 2.0 & 2.0 \\
\hline
\end{tabular}

Seager \& Mallén-Ornelas 2003). In particular, by fitting the transit shape, it is possible to obtain the measurement of the stellar and planetary relative radii, $r_{*}=\frac{R_{*}}{a}$ and $r_{b}=\frac{R_{b}}{a}$ (where $a$ is the semimajor axis of the orbit), the inclination of the planetary orbit with respect to the line of sight of the observer, $i$, and the time of the transit centre, $T_{0}$.

Using the JKTEBOP ${ }^{1}$ code (version 34, Southworth 2013, and references therein), we separately fitted each light curve initially setting the fitted parameters to the values published in the discovery paper. The values for each parameter were then obtained through a Levenberg-Marquardt minimization, while uncertainties were estimated by running Monte Carlo and residual-permutation algorithms (Southworth 2008). The coefficients of a second order polynomial were also fitted to account for instrumental and astrophysical trends possibly present in the light curves. In particular, 10000 simulations for the Monte Carlo and Ndata-1 simulations for residualpermutation algorithm were run, and the larger of the two $1 \sigma$ values were adopted as the final uncertainties. The JKTEBOP code is capable of simultaneously fitting light curves and RVs, and therefore, giving also an estimation of the semi-amplitude $K$ and systemic velocity $\gamma_{\text {sys. }}$.

To properly constrain the planetary system's quantities, we took into account the effect of the star's limb darkening (LD) while the planet is transiting the stellar disc. We applied a quadratic law to describe this effect, and used the LD coefficients provided by the stellar models of Claret $(2000,2004)$ once the stellar atmospheric parameters were supplied (Table 3). Each light curve was first fitted for the linear coefficient, while the quadratic one was perturbed during the Monte Carlo and residual-permutation algorithms in order to account for its uncertainty. Then, we repeated the fitting process while keeping both the LD coefficients fixed.

Considering the discussion in A12, we fixed the eccentricity to zero for both the planetary systems. As an extra check, we used the Systemic Console 2 (Meschiari et al. 2009) to fit the RVs published in the discovery paper and those we observed with FEROS, obtaining a value consistent with $e=0$ (from the fit we obtained $e=$ $0.041 \pm 0.043$ for WASP-45). All the light curves observed along

\footnotetext{
${ }^{1}$ The JKTEBOP source code can be downloaded at http://www.astro.
} keele.ac.uk/jkt/codes/jktebop.html 


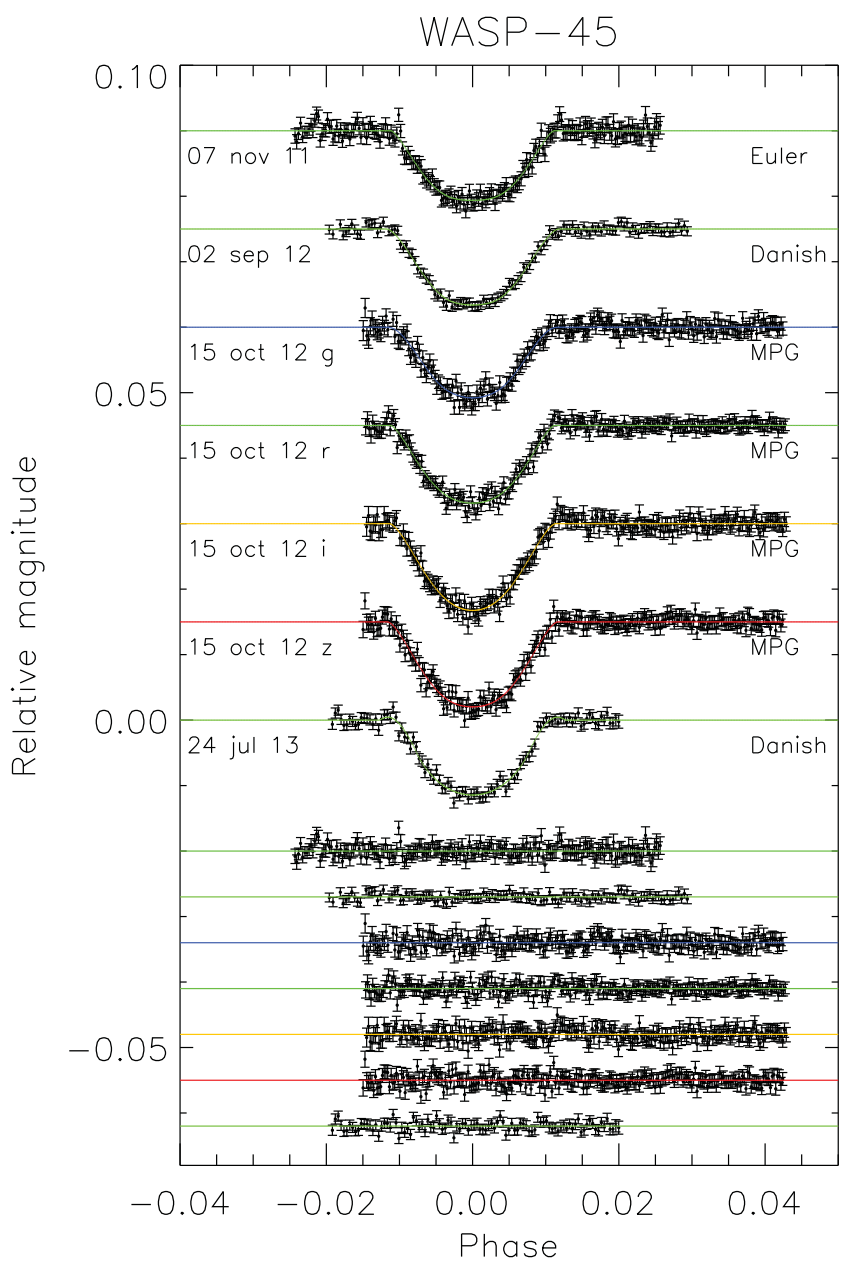

Figure 1. Phased light curves corresponding to four transits of WASP-45 b. The date and the telescope used for each transit event are indicated close to the corresponding light curve. Each light curve is shown with its best fit obtained with Jктевор. The residuals relative to each fit are displayed at the base of the figure in the same order as the light curves. The curves are shifted along the $y$-axis for clarity.

with the best fit are shown in Fig. 1 for WASP-45 and Figs 2 and 3 for WASP-46.

\subsection{New orbital ephemeris}

From the fit of each light curve, we obtained, among the other properties, accurate values of the mid-transit times. By also taking into account the values found from the discovery paper A12 and those from the Exoplanet Transit Database (ETD; Poddaný, Brát \& Pejcha 2009) ${ }^{2}$ website, we refined the ephemeris values. In particular for WASP-46, we used only those light curves from the ETD catalogue that had a data quality index better than three and whose light curve didn't show evident deviation from a transit shape that could affect the $T_{0}$ measurement. The new values for the period and the reference time of mid-transit, $T_{0}$, were obtained performing a linear fit to all the mid-transit times versus their cycle number (see Tables 4 and 5). We obtained:

$T_{0}=\mathrm{BJD}(\mathrm{TDB}) 2455441.2687(10)+3.1260960(49) E$,

\footnotetext{
${ }^{2}$ The data base can be found on http://var2.astro.cz/ETD
}

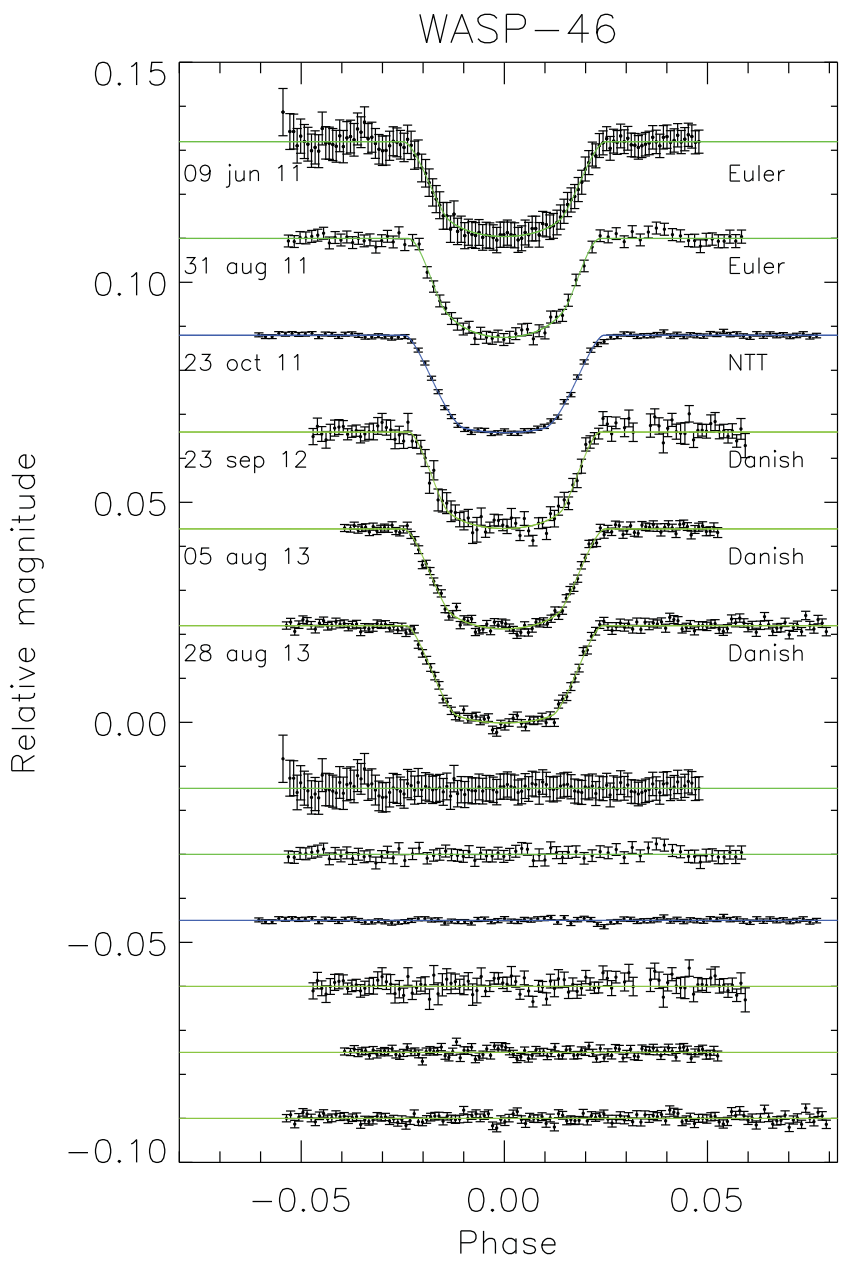

Figure 2. Phased light curves of the transits of WASP-46 b, observed with the Euler, NTT, and Danish telescopes, compared with the best-fitting curves given by JктЕвор. The date and the telescope used for each transit event are indicated close to the corresponding light curve. The residuals relative to each fit are displayed at the base of the figure in the same order as the light curves. The curves are shifted along the $y$-axis for clarity.

for WASP-45, and

$T_{0}=\mathrm{BJD}(\mathrm{TDB}) 2455392.31659(58)+1.43036763(93) E$.

for WASP-46, where the numbers in brackets represent the uncertainties on the last digit of the number they follow, and $E$ is the number of orbits the planet has completed since the $T_{0}$ used as reference. The presence of an additional planetary companion in either of the two systems can be detected thanks to the gravitational effects that it would generate on the motion of the known bodies. Indeed, if another planet orbits the same star as WASP-45 b or WASP-46 b, it will affect their orbital motion, by periodically advancing and retarding the transit time (e.g. Holman \& Murray 2005; Lissauer et al. 2011). Here, the fit has a $\chi_{v}^{2}=9.5$ and 22.7 for WASP-45 and WASP-46, respectively, indicating that the linear ephemeris is not a good match to the observations in both the cases. As noted in previous cases (e.g. Southworth et al. 2015), this is an indication that the measured timings have too small uncertainties rather than the presence of a coherent TTV. The plots of the residuals, displayed in Figs 4 and 5, do not show any evidence for systematic deviations from the predicted transit times. Indeed, by fitting the residuals with both a linear and sinusoidal function, we did not find any significative correlation or periodic signal. However, more 


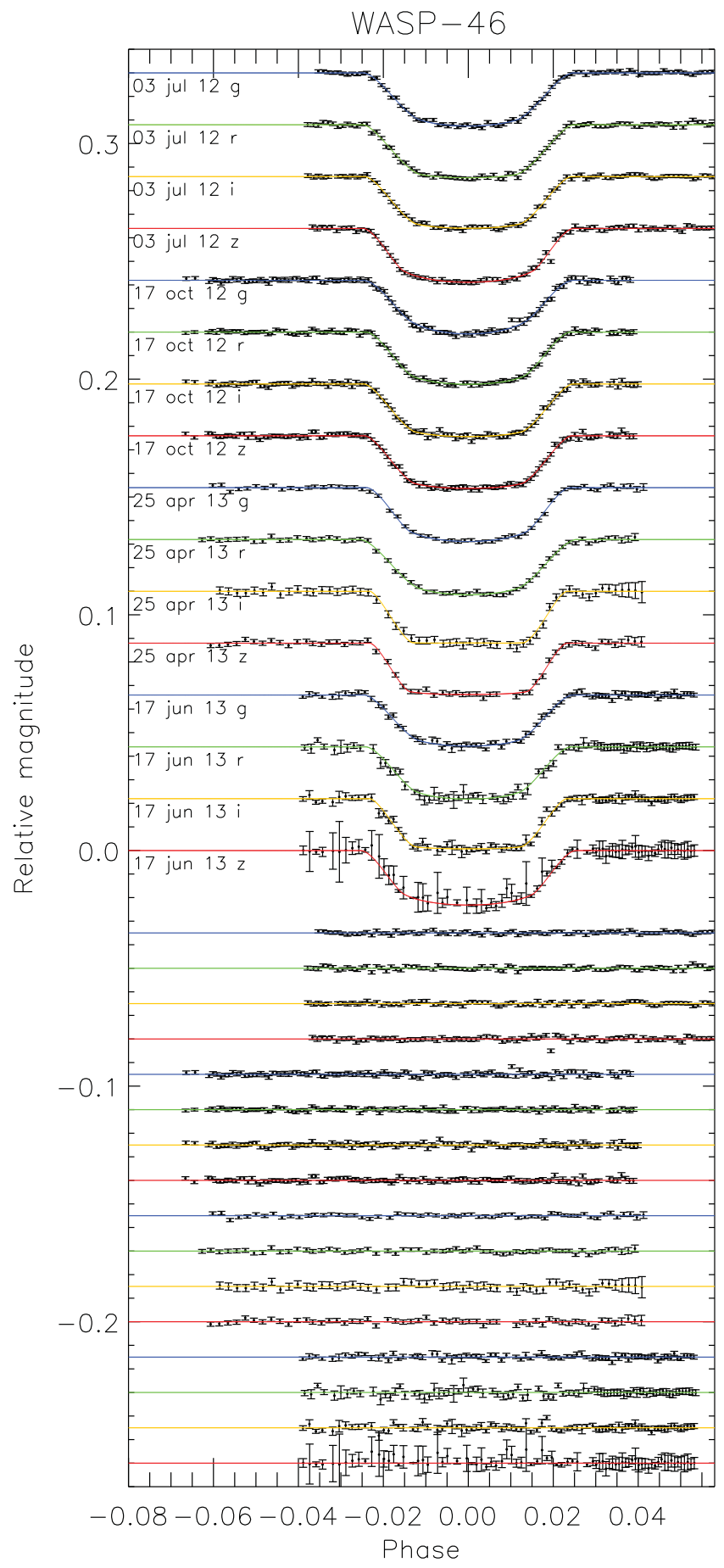

Figure 3. Phased light curves of four transits of WASP-46 observed with GROND. Each transit was observed simultaneously in four optical bands. As in Figs 1 and 2 the light curves are compared with the best-fitting curves given by ЈктЕвоР, and the residuals from the fits are displayed at the bottom in the same order as the light curves. The curves are shifted along the $y$-axis for clarity.

precise and homogenous measurements of the timings are mandatory to robustly establish the presence of a TTV in any of the two planetary systems.

A signature of additional planetary or more massive bodies in one of the two systems can also be found by looking for a periodicity or a linear trend in the residual of the RV data, once the sinusoidal signal
Table 4. Times of mid-transit point of WASP-45 $\mathrm{b}$ and their residuals. References: (1) A12; (2) ETD; (3) Euler, this work; (4) GROND, this work; (5) Danish, this work.

\begin{tabular}{lcrc}
\hline $\begin{array}{l}\text { Time of minimum } \\
\text { BJD(TDB)-2400000 }\end{array}$ & Epoch & $\begin{array}{r}\text { Residual } \\
(\text { JD })\end{array}$ & Reference \\
\hline $55441.27000 \pm 0.00058$ & 0 & -0.00128 & $(1)$ \\
$55782.01007 \pm 0.00235$ & 109 & 0.00310 & $(2)$ \\
$55872.67006 \pm 0.00030$ & 138 & -0.00011 & $(3)$ \\
$56119.62852 \pm 0.00081$ & 217 & 0.00301 & $(2)$ \\
$56172.77422 \pm 0.00018$ & 234 & 0.00094 & $(5)$ \\
$56216.54008 \pm 0.00029$ & 248 & 0.00042 & $(4) g$ \\
$56216.54153 \pm 0.00021$ & 248 & -0.00103 & $(4) r$ \\
$56216.54110 \pm 0.00024$ & 248 & -0.00060 & $(4) i$ \\
$56216.54001 \pm 0.00025$ & 248 & 0.00049 & $(4) z$ \\
$56497.88958 \pm 0.00026$ & 338 & -0.00044 & $(5)$ \\
\hline
\end{tabular}

Table 5. Times of mid-transit point of WASP-46 b and their residuals. References: (1) A12; (2) ETD; (3) Euler, this work; (4) NTT, this work; (5) GROND, this work; (6) Danish, this work.

\begin{tabular}{lrrl}
\hline $\begin{array}{l}\text { Time of minimum } \\
\text { BJD(TDB) }-2400000\end{array}$ & Epoch & $\begin{array}{r}\text { Residual } \\
(\mathrm{JD})\end{array}$ & \begin{tabular}{l} 
Reference \\
\hline $55392.31628 \pm 0.00020$
\end{tabular} \\
$55722.73197 \pm 0.00013$ & 231 & -0.00032 & $(1)$ \\
$55757.06235 \pm 0.00098$ & 255 & 0.00201 & $(3)$ \\
$55805.69409 \pm 0.00020$ & 289 & 0.00125 & $(2)$ \\
$55858.61624 \pm 0.00011$ & 326 & -0.00020 & $(4)$ \\
$56108.92758 \pm 0.00091$ & 501 & -0.00320 & $(2)$ \\
$56111.79133 \pm 0.00011$ & 503 & -0.00050 & $(5) g$ \\
$56111.79141 \pm 0.00013$ & 503 & -0.00019 & $(5) r$ \\
$56111.79132 \pm 0.00013$ & 503 & -0.00018 & $(5) i$ \\
$56111.79102 \pm 0.00013$ & 503 & -0.00010 & $(5) z$ \\
$56130.38924 \pm 0.00042$ & 516 & 0.00295 & $(2)$ \\
$56194.74962 \pm 0.00027$ & 561 & -0.00322 & $(6)$ \\
$56217.63904 \pm 0.00013$ & 577 & 0.00005 & $(5) g$ \\
$56217.63892 \pm 0.00011$ & 577 & 0.00012 & $(5) r$ \\
$56217.63883 \pm 0.00010$ & 577 & 0.00020 & $(5) i$ \\
$56217.63877 \pm 0.00012$ & 577 & 0.00032 & $(5) z$ \\
$56227.65622 \pm 0.00062$ & 584 & 0.00493 & $(2)$ \\
$56407.87778 \pm 0.00014$ & 710 & -0.00056 & $(5) g$ \\
$56407.87730 \pm 0.00033$ & 710 & -0.00051 & $(5) r$ \\
$56407.87711 \pm 0.00017$ & 710 & -0.00031 & $(5) i$ \\
$56407.87705 \pm 0.00021$ & 710 & 0.00017 & $(5) z$ \\
$56460.80084 \pm 0.00016$ & 747 & -0.00038 & $(5) g$ \\
$56460.80090 \pm 0.00020$ & 747 & -0.00031 & $(5) r$ \\
$56460.80147 \pm 0.00028$ & 747 & -0.00030 & $(5) i$ \\
$56460.80092 \pm 0.00025$ & 747 & 0.00025 & $(5) z$ \\
$56510.86498 \pm 0.00013$ & 782 & 0.00090 & $(6)$ \\
$56510.86827 \pm 0.00067$ & 782 & 0.00419 & $(2)$ \\
$56520.88045 \pm 0.00067$ & 789 & 0.00379 & $(2)$ \\
$56533.74905 \pm 0.00013$ & 798 & -0.00092 & $(6)$ \\
$56882.76628 \pm 0.00065$ & 1042 & 0.00661 & $(2)$ \\
$56942.83897 \pm 0.00083$ & 1084 & 0.00386 & $(2)$ \\
\hline & & & \\
\hline
\end{tabular}

due to the known planet is removed (e.g. Butler et al. 1999; Marcy et al. 2001). Considering both the data from the discovery paper and the new ones presented in this work, we studied the distribution of the RV residuals in time for WASP-45 (shown in Fig. 6 along with the best fit), but did not find any particular trend.

This is not surprising given the low probability to find a close in companion to a hot Jupiter (e.g. Ford 2014; Izidoro et al. 2015; Mustill, Davies \& Johansen 2015). 


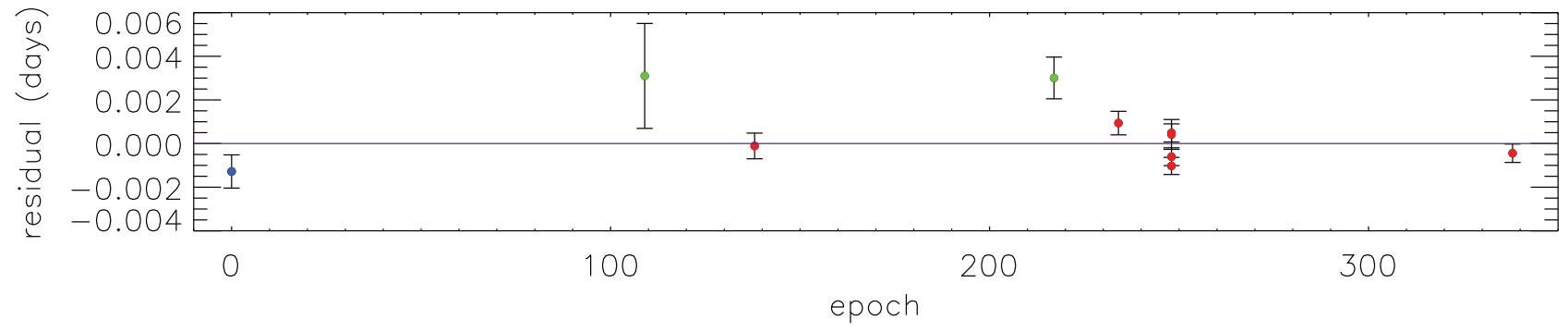

Figure 4. Plot of the residuals of the timing of mid-transit of WASP-45 versus a linear ephemeris. The different colours of the points refer to the value from the discovery paper (blue), values obtained from the ETD catalogue (green), and our data (red).

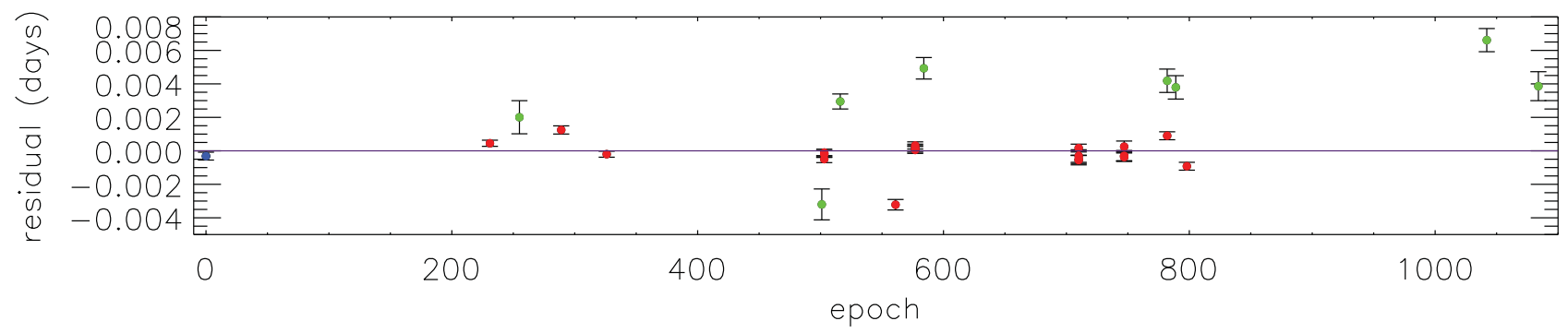

Figure 5. Plot of the residuals of the timing of mid-transit of WASP-46 versus a linear ephemeris. The different colours of the points refer to the value from the discovery paper (blue), values obtained from the ETD catalogue (green), and our data (red).
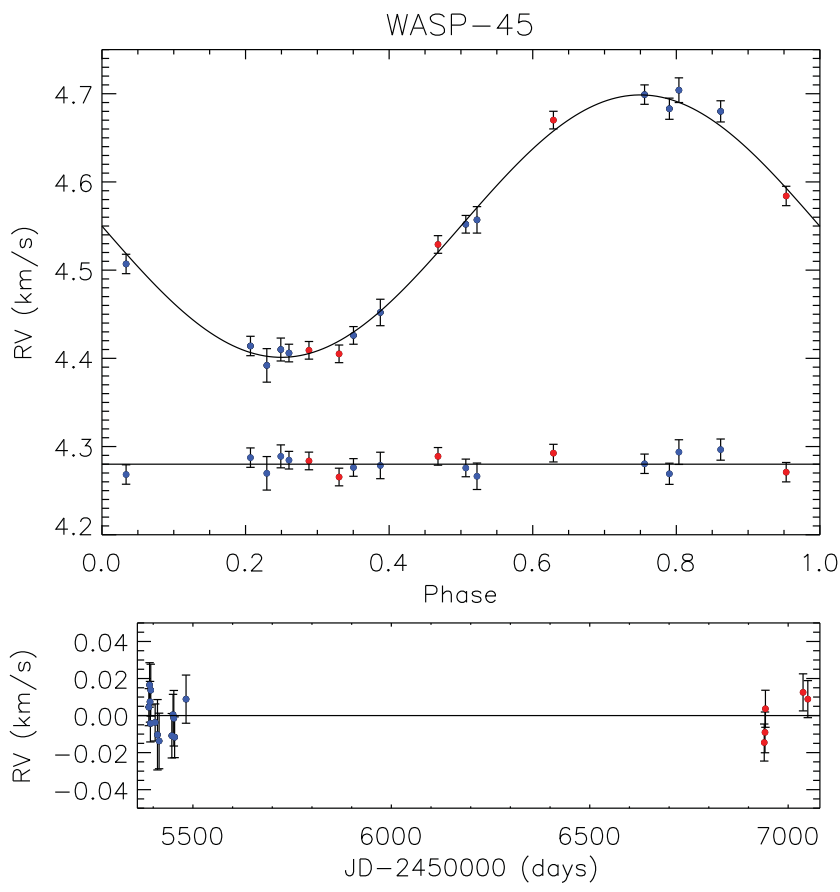

Figure 6. Upper panel: RV measurements with the best fit obtained from JKTEBOP; residuals are displayed at the bottom. Lower panel: residuals of the best fit displayed as a function of the days when the spectra were observed. In both the panels, blue points refer to the data from the discovery paper A12, while the red ones are those observed with FEROS.

\subsection{Final photometric parameters}

For both the planets, each final photometric parameter was obtained as a weighted mean of the values extracted from the fit of all the individual light curves, using the relative errors as a weight. The final uncertainties, also obtained from the weighted mean, were subsequently rescaled according to the $\sqrt{\chi_{v}^{2}}$ calculated for each quantity. The results together with their uncertainties and the relative $\chi_{v}^{2}$ are shown in Table 6 , in which they are compared with those from the discovery paper A12. The photometric parameters obtained from each single light curve are reported in Tables A1 and A2 in the Appendix.

For both planets, we decided to adopt the results obtained from the fit in which we fixed the LD coefficients for all light curves. This choice was dictated by the following reasoning. In near-grazing transits, only the region near the limb of the star is transited, so there is very little information on its LD (Howarth 2011; Müller et al. 2013). As the impact parameters of the systems are high ( $b=$ 0.87 for WASP-45 and $b=0.71$ for WASP-46), and thus the transits are nearly-grazing, we decided to not fit for the LD coefficients in order to avoid biasing the results. However, we checked that the results, obtained either fixing or fitting for the LD coefficients, were compatible with each other. We assigned to the parameters of each light curve a $1 \sigma$ uncertainty, estimated by Monte Carlo simulations, because these values were systematically higher than those obtained with the residual-permutation algorithm.

\section{PHYSICAL PROPERTIES OF WASP-45 AND WASP - 46}

Using the results obtained from the photometry (Table 6) and taking into account the spectroscopic results from the discovery paper, we redetermined the main physical parameters that characterize the two planetary systems. Following the methodology described in Southworth (2010), the missing information such as the age of the system and the planetary velocity semi-amplitude were iteratively interpolated using stellar evolutionary model predictions until the best fit to the photometric and spectroscopic parameters was reached. This was done for a sequence of ages separated by $0.1 \mathrm{Gyr}$ and covering the full main-sequence lifetimes of the stars. We independently repeated the interpolation using different stellar models (Girardi, Bressan \& Bertelli 2000; Claret 2004; Demarque et al. 2004; Pietrinferni et al. 2004; VandenBerg et al. 2006; Dotter et al. 
Table 6. Photometric properties of the WASP- 45 and WASP-46 systems derived by fitting the light curves with JKTEBOP, and taking the weighted mean of the single values obtained from each transit. The values from the discovery paper A12 are shown for comparison. $k$ is the ratio of the planetary and stellar radii, $i$ the orbital inclination, $r_{*}$ and $r_{\mathrm{b}}$ the stellar and planetary relative radii, respectively.

\begin{tabular}{|c|c|c|c|c|c|c|}
\hline \multirow[t]{2}{*}{ Parameter } & \multicolumn{3}{|c|}{ WASP-45 } & \multicolumn{3}{|c|}{ WASP-46 } \\
\hline & Result & $\chi_{v}^{2}$ & A12 & Result & $\chi_{v}^{2}$ & $\mathrm{~A} 12$ \\
\hline$r_{*}+r_{\mathrm{b}}$ & $0.1172 \pm 0.0017$ & 0.84 & $0.1217 \pm 0.0098$ & $0.1950 \pm 0.0013$ & 1.28 & $0.1992 \pm 0.0059$ \\
\hline$k$ & $0.1095 \pm 0.0024$ & 5.37 & $0.1234 \pm 0.0246$ & $0.14075 \pm 0.00035$ & 1.16 & $0.1468 \pm 0.0017$ \\
\hline$i$ (deg.) & $84.686 \pm 0.098$ & 1.03 & $84.47_{-0.79}^{+0.54}$ & $82.80 \pm 0.17$ & 1.31 & $82.63 \pm 0.38$ \\
\hline$r_{*}$ & $0.1053 \pm 0.0014$ & 0.98 & $0.1084 \pm 0.0094$ & $0.1709 \pm 0.0011$ & 1.28 & $0.1742 \pm 0.0057$ \\
\hline$r_{\mathrm{b}}$ & $0.01172 \pm 0.00026$ & 0.81 & $0.0134 \pm 0.0024$ & $0.02403 \pm 0.00021$ & 1.30 & $0.0250 \pm 0.0010$ \\
\hline
\end{tabular}

Table 7. Final results for the physical parameters of WASP-45 obtained in this work compared to those of the discovery paper. The mass $M$, radius $R$, surface gravity $g$ and mean density $\rho$ for the star and the planet are displayed; as well as the equilibrium temperature of the planet, the Safronov number $\Theta$, the semi major axis $a$ and the age of the system.

\begin{tabular}{lcc}
\hline & This work & A12 \\
\hline$M_{*}\left(\mathrm{M}_{\odot}\right)$ & $0.904 \pm 0.066 \pm 0.010$ & $0.909 \pm 0.060$ \\
$R_{*}\left(R_{\odot}\right)$ & $0.917 \pm 0.024 \pm 0.003$ & $0.945_{-0.071}^{+0.087}$ \\
$\log g_{*}(\mathrm{cgs})$ & $4.470 \pm 0.014 \pm 0.002$ & $4.445_{-0.075}^{+0.065}$ \\
$\rho_{*}\left(\rho_{\odot}\right)$ & $1.174 \pm 0.047$ & $1.08_{-0.24}^{+0.27}$ \\
$M_{\mathrm{b}}\left(M_{\text {jup }}\right)$ & $1.002 \pm 0.062 \pm 0.007$ & $1.007 \pm 0.053$ \\
$R_{\mathrm{b}}\left(R_{\text {jup }}\right)$ & $0.992 \pm 0.038 \pm 0.004$ & $1.16_{-0.14}^{+0.28}$ \\
$g_{\mathrm{b}}\left(\mathrm{ms}^{-2}\right)$ & $25.2 \pm 1.3$ & $17.0_{-6.0}^{+4.9}$ \\
$\rho_{\mathrm{b}}\left(\rho_{\text {jup }}\right)$ & $0.959 \pm 0.077 \pm 0.003$ & $0.64 \pm 0.30$ \\
$T_{\text {eq }}(\mathrm{K})$ & $1170 \pm 24$ & $1198 \pm 69$ \\
$\Theta$ & $0.0903 \pm 0.0044 \pm 0.0003$ & - \\
$a(\mathrm{au})$ & $0.0405 \pm 0.0010 \pm 0.0001$ & $0.04054 \pm 0.00090$ \\
Age $(\mathrm{Gyr})$ & $7.2_{-9.0-1.2}^{+5.8+6.8}$ & $1.4_{-1.0}^{+2.0 a}$ \\
\hline
\end{tabular}

Note. ${ }^{a}$ This is the gyrochronological age measured in A12. The same authors also obtained a value for the stellar age from lithium abundance measurements, finding that the star is at least a few Gyr old.

Table 8. Final results for the physical parameters of WASP-46 obtained in this work compared to those of the discovery paper. See Table. 7 for the description of the parameters listed. This is the gyrochronological age measured in A12.

\begin{tabular}{lcc}
\hline & This work & $\mathrm{A} 12$ \\
\hline$M_{*}\left(\mathrm{M}_{\odot}\right)$ & $0.828 \pm 0.067 \pm 0.036$ & $0.956 \pm 0.034$ \\
$R_{*}\left(R_{\odot}\right)$ & $0.858 \pm 0.024 \pm 0.013$ & $0.917 \pm 0.028$ \\
$\log g_{*}(\mathrm{cgs})$ & $4.489 \pm 0.013 \pm 0.006$ & $4.493 \pm 0.023$ \\
$\rho_{*}\left(\rho_{\odot}\right)$ & $1.310 \pm 0.025$ & $1.24 \pm 0.10$ \\
$M_{\mathrm{b}}\left(M_{\text {jup }}\right)$ & $1.91 \pm 0.11 \pm 0.06$ & $2.101 \pm 0.073$ \\
$R_{\mathrm{b}}\left(R_{\text {jup }}\right)$ & $1.174 \pm 0.033 \pm 0.017$ & $1.310 \pm 0.051$ \\
$g_{\mathrm{b}}\left(\mathrm{ms}^{-2}\right)$ & $34.3 \pm 1.1$ & $28.0_{-2.0}^{+2.2}$ \\
$\rho_{\mathrm{b}}\left(\rho_{\text {jup }}\right)$ & $1.103 \pm 0.050 \pm 0.016$ & $0.94 \pm 0.11$ \\
$T_{\mathrm{eq}}(\mathrm{K})$ & $1636 \pm 44$ & $1654 \pm 50$ \\
$\Theta$ & $0.0916 \pm 0.0035 \pm 0.0014$ & - \\
$a(\mathrm{au})$ & $0.02335 \pm 0.00063 \pm 0.00034$ & $0.02448 \pm 0.00028$ \\
Age $(\mathrm{Gyr})$ & $9.6_{-4.2-3.5}^{+3.4+1.4}$ & $1.4_{-0.6}^{+0.4 a}$ \\
\hline
\end{tabular}

Note. ${ }^{a}$ The same authors also obtained a value for the stellar age from lithium abundance measurements, finding that the star is at least a few Gyr old.

2008); for a complete list see Southworth 2010), and the final values were obtained as a weighted mean. In the final results presented in Tables 7 and 8, the first uncertainty is a statistical one, which is derived by propagating the uncertainties of the input parameters, while the second is a systematic uncertainty, which takes into account the differences in the predictions coming from the different stellar models used. The final values for the ages of the two system are not well constrained. The uncertainty that most affects the precision on these measurements is the large errorbars on $T_{\text {eff }}$ (from A12 $T_{\text {eff }}=5140 \pm 200$ and $T_{\text {eff }}=5620 \pm 160$ for WASP-45 and WASP-46, respectively). Moreover, we noticed that for metallicity different to solar, the discrepancies between the different stellar models increase, and therefore the systematic errorbar on the age estimation swells.

\section{RADIUS VERSUS WAVELENGTH VARIATION}

During a transit event, a fraction of the light coming from the host star passes through the atmosphere of the planet and, according to the atmospheric composition and opacity, it can be scattered or absorbed at specific wavelengths (Seager \& Sasselov 2000). Similarly to transmission spectroscopy, by observing a planetary transit at different bands simultaneously, it is then possible to look for variations in the value of the planet's radius measured in each band, and thus probe the composition of its atmosphere (e.g. Southworth et al. 2012; Mancini et al. 2013b; Narita et al. 2013).

To pursue this goal, we phased and binned all the light curves observed with the same instrument and filter, and performed once again a fit with JKтевор. Following Southworth et al. (2012), we fixed all the parameters to the final values previously obtained (see Tables 7 and 8) and fitted just for the planetary and stellar radii ratio $k$. In this way, we removed sources of uncertainty common to all data sets, maximizing the relative precision of the planet/star radius ratio measurements as a function of wavelength. In order to have a set of data as homogeneous as possible, we preferred to use the light curves obtained with the same reduction pipeline, and thus, we excluded the light curves from the Euler telescope from this analysis.

The values of $k$ that we obtained at different passbands are reported in Table 9 and illustrated in Figs 7 and 8 for WASP-45 and WASP-46, respectively. In these figures, for comparison, we also show the expected values of the planetary radius in function of wavelength, obtained from synthetic spectra constructed from model planetary atmospheres by Fortney et al. (2010), using different molecular compositions. The models were estimated for a Jupiter-mass planet with a surface gravity of $g_{\mathrm{p}}=25 \mathrm{~m} \mathrm{~s}^{-2}$, a base radius of $1.25 R_{\mathrm{Jup}}$ at $10 \mathrm{bar}$, and $T_{\mathrm{eq}}=1250 \mathrm{~K}$ and $1750 \mathrm{~K}$ for WASP-45 b and WASP-46 b, respectively. The model displayed with a red line in Fig. 8 was run in an isothermal case taking into account chemical equilibrium and the presence of strong absorbers, such as TiO and VO. The models displayed with blue lines in Figs 7 and 8 were obtained omitting the presence of the metal oxides.

Looking at the distribution of the experimental points in the two figures, we do not see the telltale increase of the radius at the shortest wavelengths (e.g. see Lecavelier Des Etangs et al. 2008), and therefore we do not expect a strong Rayleigh scattering in the 
Table 9. Values of $k$ for each of the light curves as plotted in Figs 7 and 8.

\begin{tabular}{lccc}
\hline Passband & $\begin{array}{c}\text { Central } \\
\text { wavelength }(\mathrm{nm})\end{array}$ & $\begin{array}{c}\text { FWHM } \\
(\mathrm{nm})\end{array}$ & $k$ \\
\hline WASP-45: & & & \\
GROND $g^{\prime}$ & 477.0 & 137.9 & $0.11090 \pm 0.00111$ \\
GROND $r^{\prime}$ & 623.1 & 138.2 & $0.11335 \pm 0.00090$ \\
Bessel $R$ & 648.9 & 164.7 & $0.11088 \pm 0.00062$ \\
Gunn $r$ & 664.1 & 85.0 & $0.10821 \pm 0.00099$ \\
GROND $i^{\prime}$ & 762.5 & 153.5 & $0.11598 \pm 0.00103$ \\
GROND $z^{\prime}$ & 913.4 & 137.0 & $0.11513 \pm 0.00089$ \\
WASP-46: & & & \\
GROND $g^{\prime}$ & 477.0 & 137.9 & $0.13950 \pm 0.00031$ \\
Gunn $g$ & 516.9 & 77.6 & $0.13961 \pm 0.00047$ \\
GROND $r^{\prime}$ & 623.1 & 138.2 & $0.13943 \pm 0.00032$ \\
Bessel $R$ & 648.9 & 164.7 & $0.13990 \pm 0.00043$ \\
Gunn $r$ & 664.1 & 85.0 & $0.13815 \pm 0.00113$ \\
GROND $i^{\prime}$ & 762.5 & 153.5 & $0.13871 \pm 0.00039$ \\
GROND $z^{\prime}$ & 913.4 & 137.0 & $0.14059 \pm 0.00042$ \\
\hline
\end{tabular}

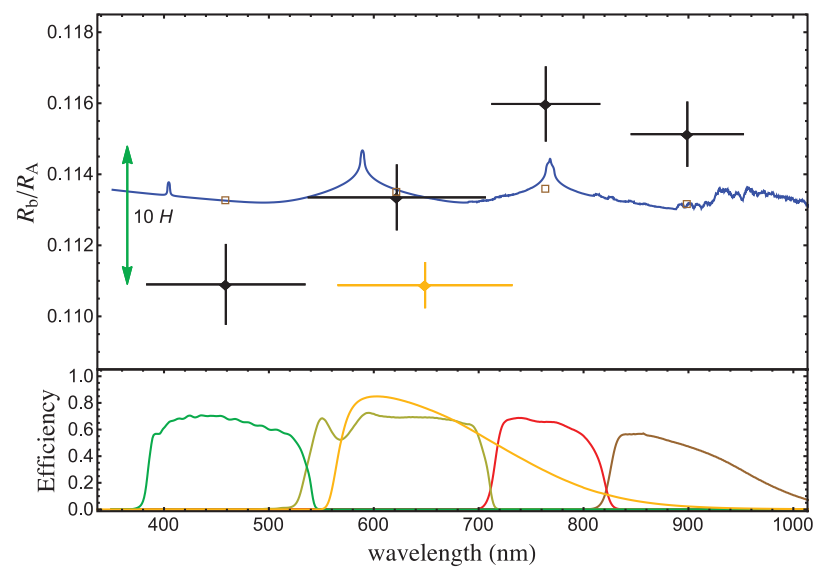

Figure 7. Variation of the radius of WASP-45 b, in terms of planet/star radius ratio, with wavelength. The black points are from the transit observed with GROND, and the yellow point is the weighted-mean results coming from the two transits observed with the Danish telescope. The vertical bars represent the uncertainties in the measurements and the horizontal bars show the FWHM transmission of the passbands used. The observational points are compared to a synthetic spectrum for a Jupiter-mass planet with a surface gravity of $g_{\mathrm{p}}=25 \mathrm{~m} \mathrm{~s}^{-2}$ and $T_{\mathrm{eq}}=1250 \mathrm{~K}$. An offset was applied to the model to provide the best fit to our radius measurements. Transmission curves of the filters used are shown in the bottom panel. On the left of the plot, the size of ten atmospheric pressure scale heights $(10 H)$ is shown. The small coloured squares represent band-averaged model radii over the bandpasses used in the observations.

atmosphere. However, by studying our data points quantitatively, we can not exclude any hypothesis. By performing a Monte Carlo simulation, we obtained that our data points are consistent within $3 \sigma$ to a slope with a maximum inclination of $m=-1.40 \times 10^{-5}$ for WASP-45 $\mathrm{b}$ and $m=-1.17 \times 10^{-5}$ for WASP-46 $\mathrm{b}$ (where with $m$ we indicate the slope coefficient of the best linear fit). Fitting with a straight line the predictions given at short wavelengths by a model with the Rayleigh scattering enhanced by a factor of 1000 , we obtained slope coefficients lower that the ones just mentioned (the slope coefficient is $m=-2.2 \times 10^{-6}$, and $m=-3.3 \times 10^{-6}$ for WASP-45 and WASP-46, respectively). Although pointing in the direction of no strong Rayleigh scattering, our data are not sufficient

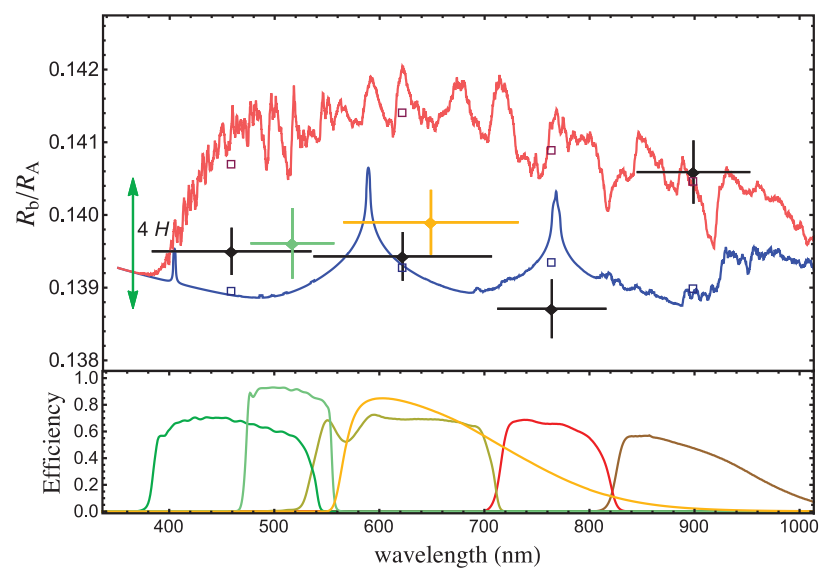

Figure 8. Variation of the radius of WASP-46 b, in terms of planet/star radius ratio, with wavelength. The black points are from the transit observed with GROND, the green point is that obtained with the NTT, and the yellow point is the weighted-mean result coming from the three transits observed with the Danish telescope. The vertical bars represent the uncertainties in the measurements and the horizontal bars show the FWHM transmission of the passbands used. The observational points are compared to two synthetic spectra for a Jupiter-mass planet with a surface gravity of $g_{\mathrm{p}}=25 \mathrm{~m} \mathrm{~s}^{-2}$, and $T_{\text {eq }}=1750 \mathrm{~K}$. The synthetic spectrum in blue does not include TiO and VO opacity, while the spectrum in red does, based on equilibrium chemistry. An offset was applied to the models to provide the best fit to our radius measurements. Transmission curves of the filters used are shown in the bottom panel. On the left of the plot, the size of four atmospheric pressure scale heights $(4 H)$ is shown. The small coloured squares represent band-averaged model radii over the bandpasses used in the observations.

to completely rule out this scenario. More data points are needed to make stronger statements regarding this matter.

For the case of WASP-45 b, for which we have only one transit observed with GROND, it is possible to note a radius variation between the $g^{\prime}$ and $i^{\prime}$ bands at $2 \sigma$, corresponding to roughly $12 H$ pressure scale heights (where the atmospheric pressure scale height is $H=k \mathrm{~T}_{\mathrm{eq}} / \mu g$, with $k$ being the Boltzmann constant, $\mathrm{T}_{\mathrm{eq}}$ the planetary atmosphere temperature, $\mu$ the mean molecular weight and $g$ the planet's surface gravity). In the case of WASP-46 b, for which we observed four transits with GROND, we noticed a small variation of $\sim 4 H$ between the $i^{\prime}$ and $z^{\prime}$ bands but at only $1.5 \sigma$. These detections are too small to be significant - both planets are not well suited to transmission photometry or spectroscopy due to their large impact parameters and high surface gravities.

As stated in A12, the lightcurve of WASP-46 shows a rotational modulation, which is symptomatic of stellar activity. The presence of star spots on the stellar surface, and in general stellar activity, can produce variations in the transit depth when it is measured at different epochs. In particular, we expected that such a variation is stronger at bluer wavelengths and affecting more the light curves obtained through the $g^{\prime}$ band, whereas it is negligible in the $i$ and $z$ bands (e.g. Sing et al. 2011; Mancini et al. 2014). Correcting for this effect, would slightly shift the data point relative to the bluer bands, towards the bottom of Fig. 7. Anyway, since the stellar activity is not particularly high, the expected variation in the transit depth is small and within our errorbars (Fig. 9 shows the effect of the presence of starspots, at different temperatures, on the transit depth with wavelength. The stellar model used to produce the curves are the ATLAS9 by Castelli \& Kurucz 2004). 


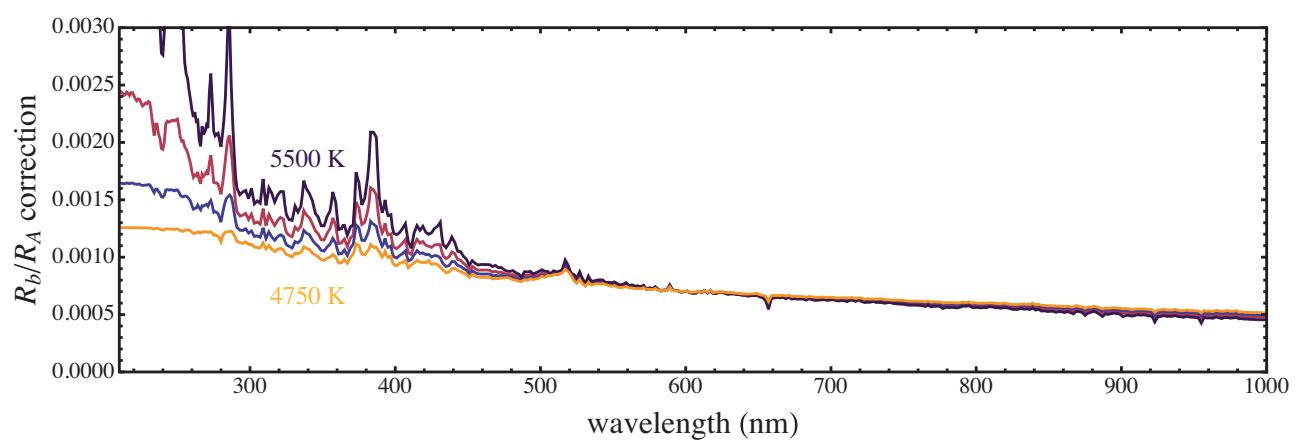

Figure 9. The effect of the presence of unocculted starspot on the surface of the star on the transmission spectrum, considering a 1 per cent flux drop at $600 \mathrm{~nm}$. The stellar temperature adopted is $T_{\text {eff }}=5600 \mathrm{~K}$, and the spots coverage is modelled using a grid of stellar atmospheric models of different temperature ranging from 4750 (yellow line) to 5500 (purple line), with steps of $250 \mathrm{~K}$.

\section{SUMMARY AND CONCLUSIONS}

We have presented new multiband photometric light curves of transit events of the hot-Jupiter planets WASP-45 b and WASP-46 b, and new RV measurements of WASP-45. We used these new data sets to refine the orbital and physical parameters that characterize the architecture of the WASP-45 and WASP-46 planetary systems. Moreover, we used the light curves observed through several optical passbands to probe the atmosphere of the two planets. Our conclusions are as follows.

(i) The radius of WASP- $45 \mathrm{~b}$ is now much better constrained, with a precision better by almost one order of magnitude with respect to that of A12. We found that WASP-46 b has a slightly lower radius $\left(1.189 \pm 0.037 R_{\text {Jup }}\right)$ compared to the previous estimation $(1.310 \pm$ $\left.0.051 R_{\mathrm{Jup}}\right)$. The left-hand panel of Fig. 10 shows the change in position in the planet mass-radius diagram for both WASP-45 $\mathrm{b}$ and WASP-46 b.

(ii) Based on our estimates, both planets have a larger density than previously thought (see the right panel of Fig. 10). In particular, WASP-45 b appears to be one of the densest planets in its mass regime (there are only three other planets with masses between 0.7 and $1.3 M_{\text {Jup }}$ that have similar or higher density), suggesting the presence of a heavy-element core of roughly $50 M_{\oplus}$ (Fortney et al. 2007).

(iii) By studying the transit times and the RV residuals, we did not find any hint for the presence of any additional planetary companion in either of the two planetary systems. However, more spectroscopic and photometric data are necessary to claim a lack of other planetary companions, at any mass and separation, in these two systems. In particular, a higher temporal cadence for the photometric observa-
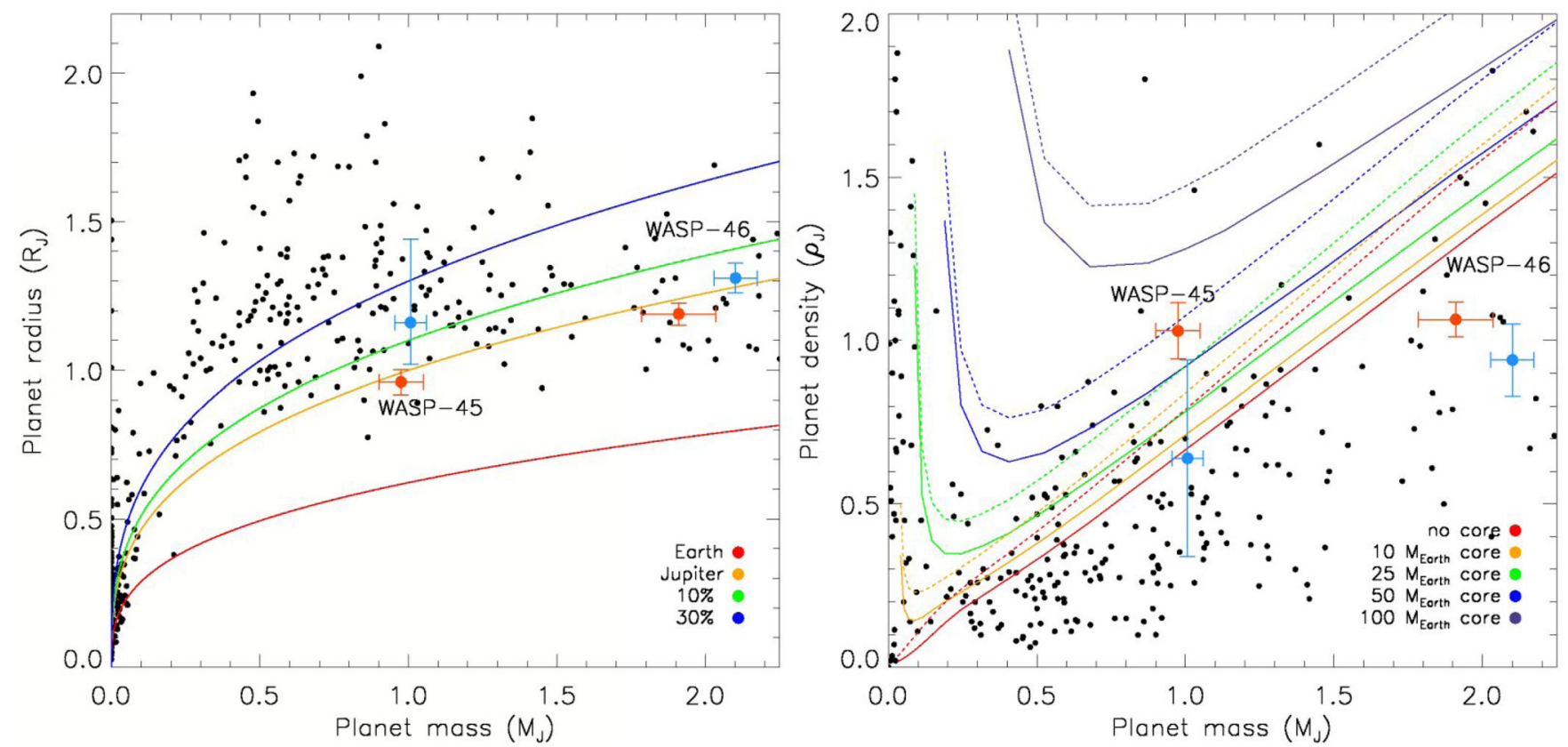

Figure 10. Left: the mass versus radius diagram of the known transiting planets. The values for WASP- $45 \mathrm{~b}$ and WASP- $46 \mathrm{~b}$ obtained in this work are displayed in orange, and for comparison we also show in light blue the measurements reported in the discovery paper. The coloured curves represent the iso-density lines for planets with the density of Earth and Jupiter, and with a density equal to a Jupiter-like planet with a radius inflated by $10-30$ per cent. Right: the mass versus density diagram of the known transiting planets. As in the left panel, the values obtained for WASP-45 and WASP-46 are highlighted in colour. The superimposed lines represent the expected radius of the planet having an inner core of 0, 10, 25, 50 and 100 Earth masses, and calculated for $10 \mathrm{Gyr}$ old planets at $0.02 \mathrm{au}$, solid lines, and $0.045 \mathrm{au}$, dashed lines (Fortney, Marley \& Barnes 2007). 
tions could allow us to detect the signature of smaller inner planets, while RV measurements obtained at different epochs separated by several months/years could provide information on the presence of outer long period planets.

(iv) Looking at the radius variation in terms of wavelength, we estimated the upper limit of the slope allowed by our data within $3 \sigma$. The slope of the best linear fit are $m=-1.40 \times 10^{-5}$ and $m=-1.17 \times 10^{-5}$ for WASP-45 b and WASP-46 b, respectively. Comparing these values to the slope obtained from a model with 1000 times enhancement of Rayleigh scattering, we found that we can not exclude with a high statistical significance the presence of strong Rayleigh scattering in the atmospheres of both planets.

The data of one transit of WASP-45 b, observed simultaneously in four optical bands with GROND, indicate a planetary radius variation of more than $10 H$ between the $g^{\prime}$ and the $i^{\prime}$ bands, but at only a $\sim 2 \sigma$ significance level. Such a variation is rather high for that expected for a planet with a temperature below $1200 \mathrm{~K}$, and would require the presence of strong absorbers between 800 and $900 \mathrm{~nm}$. More observations are requested to verify this possible scenario.

In the case of WASP-46 b, by joining the GROND multicolour data of four transit events, we detected a very small radius variation, roughly $4 H$, between the $i^{\prime}$ and $z^{\prime}$ bands, but at only a $1.5 \sigma$ significance level. This variation can be explained by supposing the absence of potassium at $770 \mathrm{~nm}$ and a significant amount of water vapour around $920 \mathrm{~nm}$.

\section{ACKNOWLEDGEMENTS}

Based on data collected by the MiNDSTEp collaboration with the Danish $1.54 \mathrm{~m}$ telescope, and on data observed with the NTT (under program number 088.C-0204(A)), $2.2 \mathrm{~m}$ and Euler-Swiss Telescope all located at the ESO La Silla Observatory.We acknowledge the use of the NASA Astrophysics Data System; the SIMBAD data base operated at CDS, Strasbourg, France; and the arXiv scientific paper preprint service operated by Cornell University. This work was supported by KASI (Korea Astronomy and Space Science Institute) grants 2012-1-410-02 and 2013-9-400-00. ASB acknowledges support from the European Union Seventh Framework Programme (FP7/2007-2013) under grant agreement number 313014 (ETAEARTH). TCH acknowledges financial support from the Korea Research Council for Fundamental Science and Technology (KRCF) through the Young Research Scientist Fellowship Program. YD, AE, JSurdej and OW acknowledge support from the Communauté française de Belgique - Actions de recherche concertées - Académie Wallonie-Europe. SHG and XBW would like to thank the financial support from National Natural Science Foundation of China through grants nos. 10873031 and 11473066. SC thanks G-D. Marleau for useful discussion and comments, the staff and astronomers observing at the ESO La Silla observatory during January and February 2015 for the great, friendly and scientifically stimulating environment.

\section{REFERENCES}

Alonso R. et al., 2004, ApJ, 613, 153

Alsubai K. A. et al., 2013, AcA, 63, 465

Anderson D. R. et al., 2012, MNRAS, 422, 1988 (A12)

Bakos G. Á., Noyes R. W., Kovács G., Stanek K. Z., Sasselov D. D., Domsa I., 2004, PASP, 116, 266

Bakos G. Á. et al., 2013, PASP, 125, 154
Baraffe I., Chabrier G., Fortney J., 2014, in Henrik B., Ralf S. Klessen, Cornelis P. D., Thomas H., eds, Protostar and Planet VI. Univ. Arizona Press, Tuscan, AZ, p. 763

Barge P. et al., 2008, A\&A, 482, L17

Baruteau C. et al., 2014, in Beuther H., Dullemond C. P., Klessen R. S., Henning T. K., eds, Protostar and Planet VI. Univ. Arizona Press, Tuscan, AZ, p. 667

Borucki W. J. et al., 2011, ApJ, 728, 117

Butler R., Marcy G. W., Fischer D. A., Brown T. M., Contos A. R., Korzennik S. G., Nisenson P., Noyes R. W., 1999, ApJ, 526, 916

Castelli F., Kurucz R. L., 2004, preprint (arXiv:astro-ph/0405087)

Charbonneau D., Brown T. M., Noyes R. W., Gilliland R. L., 2002, ApJ, 568,377

Charbonneau D. et al., 2009, Nature, 462, 891

Chen G. et al., 2014a, A\&A, 563, A40

Chen G., van Boekel R., Wang H., Nikolov N., Seemann U., Henning Th., 2014b, A\&A, 567, A8

Claret A., 2000, A\&A, 363, 1081

Claret A., 2004, A\&A, 428, 1001

Demarque P., Woo J.-H., Kim Y.-C., Yi S. K., 2004, ApJS, 155, 667

Dotter A., Chaboyer B., Jevremovic D., Kostov V., Baron E., Ferguson J. W., 2008, ApJS, 178, 89

Ford E. B., 2014, Proc. Natl. Acad. Sci. India, 111, 12616

Fortney J. J., Marley M. S., Barnes J. W., 2007, ApJ, 659, 1661

Fortney J. J., Shabram M., Showman A. P., Lian Y., Freedman R. S., Marley M. S., Lewis N. K., 2010, ApJ, 709, 1396

Gillon M., Pont F., Moutou C., Bouchy F., Courbin F., Sohy S., Magain P., 2006, A\&A, 459, 249

Girardi L., Bressan A., Bertelli G., 2000, A\&AS, 141, 371

Greiner J. et al., 2008, PASP, 120, 405

Hellier C. et al., 2011, PASP, 120, 405

Holman M. J., Murray N. W., 2005, Science, 307, 1288

Howarth I. D., 2011, MNRAS, 418, 1165

Izidoro A., Raymond S. N., Morbidelli A., Hersant F., Pierens A., 2015, ApJ, 800, 22

Jones M. I., Jenkins J. S., Rojo P., Melo C. H. F., Bluhm P., 2015, A\&A, 573,3

Jordán A. et al., 2014, AJ, 148, 29

Kley W., Nelson R. P., 2012, ARA\&A., 50, 211

Kreidberg L., Bean J. L., Désert J.-M., 2014, Nature, 505, 66

Lecavelier Des Etangs A., Pont F., Vidal-Madjar A., Sing D., 2008, A\&A, 481, L83

Lendl M. et al., 2012, A\&A, 544, 72

Lendl M., Gillon M., Queloz D., Alonso R., Fumel A., Jehin E., Naef D., 2013, A\&A, 552, A2

Lendl M. et al., 2014, A\&A, 568, 81

Lissauer J. J. et al., 2011, Nature, 470, 53

McCullough P. R., Stys J. E., Valenti J. A., Fleming S. W., Janes K. A., Heasley J. N., 2005, PASP, 117, 783

Mancini L. et al., 2013a, MNRAS, 436, 2

Mancini L. et al., 2013b, A\&A, 551, A11

Mancini L. et al., 2014, MNRAS, 443, 2391

Marcy G. W., Butler R. P., Fischer D., Vogt S. S., Lissauer J. J., Eugenio J. R., 2001, ApJ, 556, 296

Marsh T. R., 1989, PASP, 101, 1032

Meschiari S., Wolf A. S., Rivera E., Laughlin G., Vogt S., Butler P., 2009, PASP, 121, 1016

Müller H. M., Huber K. F., Czesla S., Wolter U., Schmitt J. H. M. M., 2013, A\&A, 560, 112

Mustill A. J., Davies M. B., Johansen A., 2015, ApJ, 808, 14

Narita N., Nagayama T., Suenaga T., Fukui A., Ikoma M., Nakajima Y., Nishiyama S., Tamura M., 2013, PASJ, 65, 27

Penev K. et al., 2013, AJ, 145, 5

Pepper J. et al., 2007, PASP, 119, 923

Poddaný S., Brát L., Pejcha O., 2009, preprint (arXiv:0909.2548)

Pietrinferni A., Cassisi S., Salaris M., Castelli F., 2004, ApJ, 612, 168

Pollacco D. L. et al., 2006, PASP, 118, 1407 
Richardson L. J., Deming D., Wiedemann G., Goukenleuque C., Steyert D., Harrington J., Esposito L. W., 2003, ApJ, 584, 1053

Seager S., Mallén-Ornelas G., 2003, ApJ, 585, 1038

Seager S., Sasselov D. D., 2000, ApJ, 537, 916

Sing D. K. et al., 2011, MNRAS, 416, 1443

Southworth J., 2008, MNRAS, 386, 1644

Southworth J., 2010, MNRAS, 408, 1689

Southworth J., 2013, A\&A, 557, A119

Southworth J. et al., 2009, MNRAS, 396, 1023

Southworth J., Mancini L., Maxted P. F. L., Bruni I., Tregloan-Reed J., Barbieri M., Ruocco N., Wheatley P. J., 2012, MNRAS, 422, 3099

Southworth J. et al., 2014, MNRAS, 444, 776
Southworth, J. et al., 2015, MNRAS, 447, 711

Stetson P. B., 1987, PASP, 99, 191

VandenBerg D. A., Bergbusch P. A., Dowler P. D., 2006, ApJS, 162, 375

Winn J. N. et al., 2007, AJ, 134, 1707

\section{APPENDIX A: PHOTOMETRIC PARAMETERS}

In the two tables in this appendix are presented the photometric results obtained with ЈктевоP from the fit of each light curve presented in this paper.

Table A1. Photometric properties of the WASP-45 system derived by fitting the light curves with JKTEBOP. In bold are highlighted the final parameters obtained as weighted mean. The values from the discovery paper are also shown for comparison.

\begin{tabular}{|c|c|c|c|c|c|}
\hline Source & $r_{\mathrm{A}}+r_{\mathrm{b}}$ & $k$ & $i$ (deg.) & $r_{\mathrm{A}}$ & $r_{\mathrm{b}}$ \\
\hline Dan $1.54 \mathrm{~m}$ (transit no.1) & $0.1188 \pm 0.0032$ & $0.1130 \pm 0.0022$ & $84.6423 \pm 0.1809$ & $0.10671 \pm 0.0027$ & $0.012061 \pm 0.00049$ \\
\hline MPG $2.2 \mathrm{~m}$ (transit r) & $0.1140 \pm 0.0036$ & $0.1128 \pm 0.0022$ & $84.9141 \pm 0.2079$ & $0.10245 \pm 0.0030$ & $0.011558 \pm 0.00054$ \\
\hline MPG $2.2 \mathrm{~m}$ (transit i) & $0.1181 \pm 0.0061$ & $0.1234 \pm 0.0089$ & $84.6154 \pm 0.3761$ & $0.10516 \pm 0.0046$ & $0.012979 \pm 0.00149$ \\
\hline MPG $2.2 \mathrm{~m}$ (transit z) & $0.1198 \pm 0.0046$ & $0.1176 \pm 0.0035$ & $84.5609 \pm 0.2778$ & $0.10719 \pm 0.0038$ & $0.012601 \pm 0.00079$ \\
\hline Dan $1.54 \mathrm{~m}$ (transit no.2) & $0.1076 \pm 0.0060$ & $0.1097 \pm 0.0035$ & $85.2537 \pm 0.3657$ & $0.09699 \pm 0.0051$ & $0.010640 \pm 0.00088$ \\
\hline Anderson et al. (2012) & $0.1217 \pm 0.0098$ & $0.1234 \pm 0.0246$ & $84.47_{-0.79}^{+0.54}$ & $0.1084 \pm 0.0094$ & $0.0134 \pm 0.0024$ \\
\hline
\end{tabular}

Table A2. Photometric properties of the WASP-46 system derived by fitting the light curves with JKTEBOP. In bold are highlighted the final parameters obtained as weighted mean. The values from the discovery paper are also shown for comparison.

\begin{tabular}{|c|c|c|c|c|c|}
\hline Source & $r_{\mathrm{A}}+r_{\mathrm{b}}$ & $k$ & $i$ (deg.) & $r_{\mathrm{A}}$ & $r_{\mathrm{b}}$ \\
\hline Eul 1.2 m (transit no. 2) & $0.1857 \pm 0.0099$ & $0.14025 \pm 0.00296$ & $83.51 \pm 0.70$ & $0.1629 \pm 0.0084$ & $0.02284 \pm 0.00158$ \\
\hline MPG 2.2 m (transit no. $1 \mathrm{~g}$ ) & $0.1996 \pm 0.0041$ & $0.14226 \pm 0.00127$ & $82.72 \pm 0.27$ & $0.1747 \pm 0.0034$ & $0.02485 \pm 0.00068$ \\
\hline MPG $2.2 \mathrm{~m}$ (transit no. $1 \mathrm{r}$ ) & $0.1997 \pm 0.0046$ & $0.14229 \pm 0.00115$ & $82.77 \pm 0.31$ & $0.1748 \pm 0.0039$ & $0.02487 \pm 0.00072$ \\
\hline MPG $2.2 \mathrm{~m}$ (transit no. $1 \mathrm{i})$ & $0.1938 \pm 0.0042$ & $0.13991 \pm 0.00102$ & $83.05 \pm 0.30$ & $0.1700 \pm 0.0035$ & $0.02379 \pm 0.00063$ \\
\hline MPG $2.2 \mathrm{~m}$ (transit no. $2 \mathrm{~g}$ ) & $0.1971 \pm 0.0048$ & $0.14185 \pm 0.00162$ & $82.85 \pm 0.33$ & $0.1726 \pm 0.0040$ & $0.02449 \pm 0.00082$ \\
\hline MPG 2.2 m (transit no. $2 \mathrm{r}$ ) & $0.1902 \pm 0.0037$ & $0.13955 \pm 0.00108$ & $83.25 \pm 0.25$ & $0.1669 \pm 0.0031$ & $0.02329 \pm 0.00059$ \\
\hline MPG 2.2 m (transit no. 2 i) & $0.1968 \pm 0.0042$ & $0.14120 \pm 0.00117$ & $82.93 \pm 0.29$ & $0.1725 \pm 0.0036$ & $0.02435 \pm 0.00066$ \\
\hline MPG 2.2 m (transit no. $2 \mathrm{z}$ ) & $0.1953 \pm 0.0041$ & $0.14135 \pm 0.00108$ & $82.96 \pm 0.28$ & $0.1711 \pm 0.0035$ & $0.02418 \pm 0.00064$ \\
\hline MPG $2.2 \mathrm{~m}$ (transit no. $3 \mathrm{~g}$ ) & $0.1805 \pm 0.0103$ & $0.13800 \pm 0.00288$ & $84.14 \pm 0.80$ & $0.1586 \pm 0.0087$ & $0.02189 \pm 0.00163$ \\
\hline MPG $2.2 \mathrm{~m}$ (transit no. $3 \mathrm{r}$ ) & $0.2067 \pm 0.0053$ & $0.14525 \pm 0.00169$ & $82.25 \pm 0.33$ & $0.1805 \pm 0.0044$ & $0.02621 \pm 0.00091$ \\
\hline MPG $2.2 \mathrm{~m}$ (transit no. $4 \mathrm{z}$ ) & $0.1945 \pm 0.0090$ & $0.14015 \pm 0.00298$ & $83.30 \pm 0.69$ & $0.1706 \pm 0.0075$ & $0.02391 \pm 0.00149$ \\
\hline Dan 1.54 m (transit no. 2) & $0.1959 \pm 0.0046$ & $0.14088 \pm 0.00153$ & $82.98 \pm 0.31$ & $0.1717 \pm 0.0038$ & $0.02419 \pm 0.00076$ \\
\hline Dan 1.54 m (transit no. 3) & $0.1930 \pm 0.0049$ & $0.14039 \pm 0.00136$ & $83.09 \pm 0.34$ & $0.1692 \pm 0.0042$ & $0.02376 \pm 0.00078$ \\
\hline Final results & $\mathbf{0 . 1 9 5 0} \pm \mathbf{0 . 0 0 1 3}$ & $0.14075 \pm 0.00035$ & $82.80 \pm 0.17$ & $0.1709 \pm 0.0011$ & $0.02403 \pm 0.00021$ \\
\hline Anderson et al. (2012) & $0.1992 \pm 0.0059$ & $0.1468 \pm 0.0017$ & $82.63 \pm 0.38$ & $0.1742 \pm 0.0057$ & $0.0250 \pm 0.0010$ \\
\hline
\end{tabular}


${ }^{1}$ Max Planck Institute for Astronomy, Königstuhl 17, D-69117-Heidelberg, Germany

${ }^{2}$ INAF - Osservatorio Astrofisico di Torino, via Osservatorio 20, I-10025 Pino Torinese, Italy

${ }^{3}$ Astrophysics Group, Keele University, Staffordshire, ST5 5BG, UK

${ }^{4}$ Austrian Academy of Sciences, Space Research Institute, Schmiedlstrasse 6, A-8042 Graz, Austria

${ }^{5}$ Observatoire de Genève, Université de Genève, Chemin des maillettes 51, CH-1290 Sauverny, Switzerland

${ }^{6}$ NASA Ames Research Center, Moffett Field, CA 94035, USA

${ }^{7}$ Instituto de Astrofísica, Pontificia Universidad Católica de Chile, Av. Vicuña Mackenna 4860, 7820436 - Macul, Santiago, Chile

${ }^{8}$ Millennium Institute of Astrophysics, Av. Vicuña Mackenna 4860, 7820436

- Macul, Santiago, Chile

${ }^{9}$ Key Laboratory of Planetary Sciences, Purple Mountain Observatory, Chinese Academy of Sciences, Nanjing 210008, China

${ }^{10}$ Instituto de Astrofísica de Canarias, E-38205 La Laguna, Tenerife, Spain

${ }^{11}$ International Institute for Advanced Scientific Studies (IIASS), Via G. Pellegrino 19, I-84019 - Vietri Sul Mare (SA), Italy

${ }^{12}$ Department of Physics 'E. R. Caianiello', University of Salerno, Via Giovanni Paolo II 132, Fisciano (SA) - 84084

${ }^{13}$ Istituto Nazionale di Fisica Nucleare, Sezione di Napoli, I-80126 Napoli, Italy

${ }^{14}$ SUPA, University of St Andrews, School of Physics \& Astronomy, North Haugh, St Andrews, Fife KY16 9SS, UK

${ }^{15}$ European Southern Observatory, Karl-Schwarzschild Straße 2, D-85748

- Garching bei München, Germany

${ }^{16}$ Niels Bohr Institute \& Centre for Star and Planet Formation, University of Copenhagen, Øster Voldgade 5, DK-1350 - Copenhagen K, Denmark

${ }^{17}$ Korea Astronomy and Space Science Institute, 305-348 Daejeon, Republic of Korea

${ }^{18}$ Finnish Centre for Astronomy with ESO (FINCA), University of Turku, Väisäläntie 20, FI-21500 Piikkiö, Finland

${ }^{19}$ School of Physics, UNSW Australia, NSW 2052, Australia

${ }^{20}$ Australian Centre for Astrobiology, UNSW Australia, NSW 2052, Australia
${ }^{21}$ Qatar Environment and Energy Research Institute, Qatar Foundation, Tornado Tower, Floor 19, PO Box 5825, Doha, Qatar

${ }^{22}$ Department of Astronomy, Boston University, 725 Commonwealth Avenue, Boston, MA 02215, USA

${ }^{23}$ NASA Exoplanet Science Institute, MS 100-22, California Institute of Technology, Pasadena CA 91125

${ }^{24}$ Institut d'Astrophysique et de Géophysique, Université de Liège, Allée du 6 Août 17, Bât. B5C, Liège 1, B-4000 Belgium

${ }^{25}$ Astronomisches Rechen-Institut, Zentrum für Astronomie, Universität Heidelberg, Mnchhofstraße 12-14, D-69120 Heidelberg, Germany

${ }^{26}$ Hamburger Sternwarte, Universität Hamburg, Gojenbergsweg 112, D21029 Hamburg, Germany

${ }^{27}$ Dipartimento di Fisica e Astronomia, Universit di Bologna, Viale Berti Pichat 6/2, I-40127 Bologna, Italy

${ }^{28}$ Main Astronomical Observatory, Academy of Sciences of Ukraine, vul. Akademika Zabolotnoho 27, UA-03680 Kyiv, Ukraine

${ }^{29}$ Yunnan Observatories, Chinese Academy of Sciences, Kunming 650011, China

${ }^{30}$ Key Laboratory for the Structure and Evolution of Celestial Objects, Chinese Academy of Sciences, Kunming 650011, China

${ }^{31}$ Space Telescope Science Institute, 3700 San Martin Drive, Baltimore, MD 21218, USA

${ }^{32}$ Department of Astronomy, Ohio State University, McPherson Laboratory, 140 West 18th Avenue, Columbus, OH 43210-1173, USA

${ }^{33}$ Department of Physics, Sharif University of Technology, PO Box 11155 9161 Tehran, Iran

${ }^{34}$ Centre for Electronic Imaging, Dept. of Physical Sciences, The Open University, Milton Keynes MK7 6AA, UK

${ }^{35}$ Planetary and Space Sciences, Department of Physical Sciences, The Open University, Milton Keynes, MK7 6AA, UK

${ }^{36}$ Max Planck Institute for Solar System Research, Justus-von-Liebig-Weg 3, D-37077 Göttingen, Germany

This paper has been typeset from a $\mathrm{T}_{\mathrm{E}} \mathrm{X} / \mathrm{L} \mathrm{T}_{\mathrm{E}} \mathrm{X}$ file prepared by the author. 